\title{
Polarimetry at millimeter wavelengths with the NIKA camera: calibration and performance
}

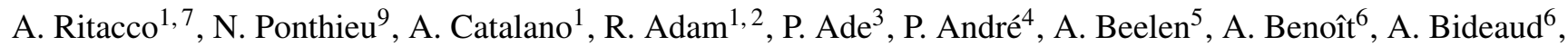 \\ N. Billot ${ }^{7}$, O. Bourrion ${ }^{1}$, M. Calvo ${ }^{6}$, G. Coiffard ${ }^{8}$, B. Comis ${ }^{1}$, F.-X. Désert ${ }^{9}$, S. Doyle ${ }^{3}$, J. Goupy ${ }^{6}$, C. Kramer ${ }^{7}$, \\ S. Leclercq ${ }^{8}$, J. F. Macías-Pérez ${ }^{1}$, P. Mauskopf ${ }^{3,10}$, A. Maury ${ }^{4}$, F. Mayet ${ }^{1}$, A. Monfardini ${ }^{6}$, F. Pajot ${ }^{5}$, E. Pascale ${ }^{3}$, \\ L. Perotto ${ }^{1}$, G. Pisano ${ }^{3}$, M. Rebolo-Iglesias ${ }^{1}$, V. Revéret ${ }^{4}$, L. Rodriguez ${ }^{4}$, C. Romero ${ }^{8}$, F. Ruppin ${ }^{1}$, G. Savini ${ }^{11}$,

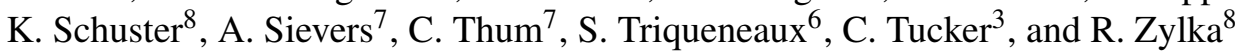

${ }^{1}$ Laboratoire de Physique Subatomique et de Cosmologie, Université Grenoble Alpes, CNRS/IN2P3, 53 avenue des Martyrs, 38000 Grenoble, France

2 Laboratoire Lagrange, Université Côte d'Azur, Observatoire de la Côte d'Azur, CNRS, Bvd de l'Observatoire, CS 34229, 06304 Nice Cedex 4, France

3 Astronomy Instrumentation Group, University of Cardiff, Cardiff Wales CF10 3AT, UK

${ }^{4}$ Laboratoire AIM, CEA/IRFU, CNRS/INSU, Université Paris Diderot, CEA-Saclay, 91191 Gif-Sur-Yvette, France

5 Institut d'Astrophysique Spatiale (IAS), CNRS and Université Paris Sud, 91405 Orsay, France

${ }^{6}$ Institut Néel, CNRS and Université Grenoble Alpes, 38000 Grenoble, France

7 Institut de RadioAstronomie Millimétrique (IRAM), 18012 Granada, Spain e-mail: ritaccoa@iram.es

8 Institut de RadioAstronomie Millimétrique (IRAM), 38000 Grenoble, France

9 Univ. Grenoble Alpes, CNRS, IPAG, 38000 Grenoble, France

${ }^{10}$ School of Earth and Space Exploration and Department of Physics, Arizona State University, Tempe, AZ 85287, USA

11 University College London, Department of Physics and Astronomy, Gower Street, London WC1E 6BT, UK

Received 7 September 2016 / Accepted 3 December 2016

\begin{abstract}
Magnetic fields, which play a major role in a large number of astrophysical processes can be traced via observations of dust polarization. In particular, Planck low-resolution observations of dust polarization have demonstrated that Galactic filamentary structures, where star formation takes place, are associated to well organized magnetic fields. A better understanding of this process requires detailed observations of galactic dust polarization on scales of 0.01 to $0.1 \mathrm{pc}$. Such high-resolution polarization observations can be carried out at the IRAM $30 \mathrm{~m}$ telescope using the recently installed NIKA2 camera, which features two frequency bands at 260 and $150 \mathrm{GHz}$ (respectively 1.15 and $2.05 \mathrm{~mm}$ ), the $260 \mathrm{GHz}$ band being polarization sensitive. NIKA2 so far in commissioning phase, has its focal plane filled with $\sim 3300$ detectors to cover a Field of View (FoV) of 6.5 arcmin diameter. The NIKA camera, which consisted of two arrays of 132 and 224 Lumped Element Kinetic Inductance Detectors (LEKIDs) and a FWHM (Full-Width-Half-Maximum) of 12 and 18.2 arcsec at 1.15 and $2.05 \mathrm{~mm}$ respectively, has been operated at the IRAM $30 \mathrm{~m}$ telescope from 2012 to 2015 as a test-bench for NIKA2. NIKA was equipped of a room temperature polarization system (a half wave plate (HWP) and a grid polarizer facing the NIKA cryostat window). The fast and continuous rotation of the HWP permits the quasi simultaneous reconstruction of the three Stokes parameters, $I, Q$, and $U$ at 150 and $260 \mathrm{GHz}$. This paper presents the first polarization measurements with KIDs and reports the polarization performance of the NIKA camera and the pertinence of the choice of the polarization setup in the perspective of NIKA2. We describe the polarized data reduction pipeline, specifically developed for this project and how the continuous rotation of the HWP permits to shift the polarized signal far from any low frequency noise. We also present the dedicated algorithm developed to correct systematic leakage effects. We report results on compact and extended sources obtained during the February 2015 technical campaign. These results demonstrate a good understanding of polarization systematics and state-of-the-art performance in terms of photometry, polarization degree and polarization angle reconstruction.
\end{abstract}

Key words. ISM: magnetic fields - polarization - ISM: clouds - instrumentation: polarimeters

\section{Introduction}

Magnetic fields have been proven to play a predominant role in a large number of astrophysical processes from galactic to cosmological scales. In particular, recent observations obtained with Herschel and Planck (Planck Collaboration I 2014) satellites have provided us with sensitive maps of the starforming complexes in the galaxy. These maps reveal large-scale filamentary structures as the preferential sites of star formation (Molinari et al. 2010; Arzoumanian et al. 2011). These filamentary structures are associated with organized magnetic field topology at scales larger than $0.5 \mathrm{pc}$ (André et al. 2014) and indicate that magnetic field must be explored on scales of 0.01 to $0.1 \mathrm{pc}$ (Pereyra \& Magalhães 2004; Planck Collaboration Int. XXXIII 2016). At millimeter and sub-millimeter wavelengths, the magnetic field orientation can be explored using 
the polarized thermal dust emission (Planck Collaboration XIX 2015; Planck Collaboration Int. XLII 2016). Dust grains are generally prolate. The polarization emission of a grain depends on the orientation and acceleration of its magnetic dipole moment and is stronger along the major axis of the grain that aligns orthogonally to the magnetic field (Lazarian 2009). This results in coherently polarized dust emission in the plane perpendicular to the magnetic field lines. Thus, polarized dust emission permits us to recover the direction of the magnetic field lines projected on the plane of the sky (e.g., Planck Collaboration XXI 2015). The Planck satellite has mapped the polarized dust emission at $353 \mathrm{GHz}$ on large angular scales over the entire sky (Planck Collaboration VIII 2014; Planck Collaboration XIII 2015) and suggests a high degree of polarization, up to $15 \%$ (Planck Collaboration XIX 2015), confirming previous Archeops results (Benoit et al. 2004). This opens a new window on the understanding of galactic magnetic fields.

Unfortunately, the 5 arcmin resolution of the Planck $353 \mathrm{GHz}$ data limits the study of the galactic magnetic field at scales of 0.2 to $0.5 \mathrm{pc}$ even for the closest clouds. For a detailed exploration of the magnetic field lines in the star-forming filamentary structures we need to perform high-resolution observations (10-20 arcsec resolution) of the polarized dust emission (Zhang et al. 2014).

The NIKA2 dual-band millimeter camera (Calvo et al. 2016; Catalano et al. 2016), recently (October 2015) installed at the IRAM 30 m telescope in Pico Veleta (Spain), is particularly well adapted to such high-resolution observations of the polarized thermal dust emission. NIKA2 features two frequency bands at 260 (polarized) and 150 (non polarized) $\mathrm{GHz}$ for a total of 3300 Lumped Element Kinetic Inductance Detectors (LEKIDs). NIKA2 has expected to have 12 (resp. 18.2) arcsec Full Width Half Maximum (FWHM) resolution at $260 \mathrm{GHz}$ (resp. $150 \mathrm{GHz}$ ) and a 6.5 arcmin diameter Field of View (FoV) at both frequencies. Between 2012 and 2015, a prototype version of NIKA2 named NIKA (Monfardini et al. 2010; Catalano et al. 2014) was operated at the IRAM $30 \mathrm{~m}$ telescope as a test-bench. NIKA was also a dual-band camera at 150 and $260 \mathrm{GHz}$ with a total of 356 LEKIDs, 12 and 18.2 arcsec resolution, but a 1.8 arcmin diameter FoV. Thanks to a specifically designed polarization setup NIKA has provided polarized observations at both frequency bands (Ritacco et al. 2016). This polarization setup includes an analyzer and a half-wave plate (HWP).

Experiments such as Planck (Planck Collaboration I 2011), BICEP (Takahashi et al. 2010), ACTPol (Niemack et al. 2010), QUaD (Hinderks et al. 2009), QUIET (Hinderks et al. 2009) and QUIJOTE (Génova-Santos et al. 2015) rotate the instrument with respect to the sky. This modulates the input polarization signal providing the required angular coverage to reconstruct the $I, Q$, and $U$ Stokes parameters. By contrast, other experiments rotate a HWP in front of an analyzer to modulate the incoming sky polarization. HERTZ (Schleuning et al. 1997), SCUPOL (Greaves et al. 2003), SHARP polarimeter (Li et al. 2008), PILOT (Misawa et al. 2014), BLASTPol (Galitzki et al. 2014), SPIDER (Fraisse et al. 2013), POLARBEAR (Barron et al. 2013), and SMA (Marrone \& Rao 2008) change the HWP orientation step by step and maintain it fixed during some periods of observation. EBEX (Chapman et al. 2014), POLKA (Wiesemeyer et al. 2014), ABS (Essinger-Hileman et al. 2016), NIKA, and NIKA2 take another option, that is, to rotate the HWP continuously.

We discuss in this paper the polarization performance of the NIKA camera and the implications for the NIKA2 design. The paper is organized as follows: Sect. 2 presents the NIKA instrument and the polarization setup. Section 3 discusses the laboratory characterization of the polarization setup. Section 4 presents the observational strategy and the dedicated polarization-datareduction pipeline. Section 5 discusses observations on quasars; Sect. 6 presents the polarization maps of few extended sources, Orion OMC-1, M 87 and Cygnus A. We draw conclusions in Sect. 7.

\section{The NIKA instrument}

Extensive descriptions of NIKA and its intensity performances can be found in Adam et al. (2014), Catalano et al. (2014), Monfardini et al. (2010, 2011), Calvo et al. (2013). Adam et al. $(2015,2016)$ also provides information about NIKA performance, reporting the characterization of the processing transfer function and extra-noise induced by astrophysical contaminants. Here we give a brief summary of the instrument and focus on the extra module that was added to provide NIKA with polarimetric capabilities.

\subsection{NIKA: a dual-band LEKID camera}

NIKA is a dual-band camera consisting of two arrays filled by LEKIDs with a Hilbert geometry (Roesch et al. 2012). LEKIDs are superconducting resonators. When photons are absorbed, they break Cooper pairs in the superconducting resonant element. This changes the density of quasi-particles and modifies the kinetic inductance, and hence the resonant frequency of the LEKID (Doyle et al. 2008). The absorbed power can be directly related to the shift of the resonant frequency (Calvo et al. 2013). The two LEKID arrays were cooled down to their optimal temperature of approximately $100 \mathrm{mK}$ using a $4 \mathrm{~K}$ cryocooler combined to a closed-cycle ${ }^{3} \mathrm{He}-{ }^{4} \mathrm{He}$ dilution. The optical coupling between the telescope and the detectors was achieved by warm aluminium mirrors and cold refractive optics (Catalano et al. 2014). The camera observed the sky in two millimeter channels, at $1.15 \mathrm{~mm}$ and $2.05 \mathrm{~mm}$, corresponding to bandwidths of 220$270 \mathrm{GHz}$ and 137-172 GHz, respectively, with central frequencies of $260 \mathrm{GHz}$ and $150 \mathrm{GHz}$, respectively. NIKA had a Field of View (FoV) of approximately 1.8 arcmin and angular resolutions (FWHM) of $18.2 \operatorname{arcsec}$ and $12 \operatorname{arcsec}$ at $150 \mathrm{GHz}$ and $260 \mathrm{GHz}$, respectively (Catalano et al. 2014; Adam et al. 2014).

\subsection{The polarimetric module}

The polarization setup of the NIKA camera consisted of a continuously rotating HWP and an analyzer, at room temperature, facing the cryostat window. The Hilbert geometry (Roesch et al. 2012) of the NIKA LEKIDS was specifically optimized for intensity observations and, as a consequence, NIKA LEKIDs are not sensitive to polarization. Therefore, for polarization observations, the analyzer was placed after the HWP at a distance of $6 \mathrm{~cm}$ from the cryostat window as shown in the two configurations of Fig. 1. The analyzer, consisting of a lithographic kapton coper polarimeter, was tilted by $10 \mathrm{deg}$ with respect to the optical axis to avoid standing waves with the cold optical filters inside the cryostat. The HWP was placed before the analyzer and shared the same holder. We used a hot-pressed metal mesh HWP designed and built at Cardiff University (Pisano et al. 2006). The HWP was designed to allow an approximately constant phase shift of the transmitted radiation over a broad spectral band including the two NIKA bands. A two-layer broadband 

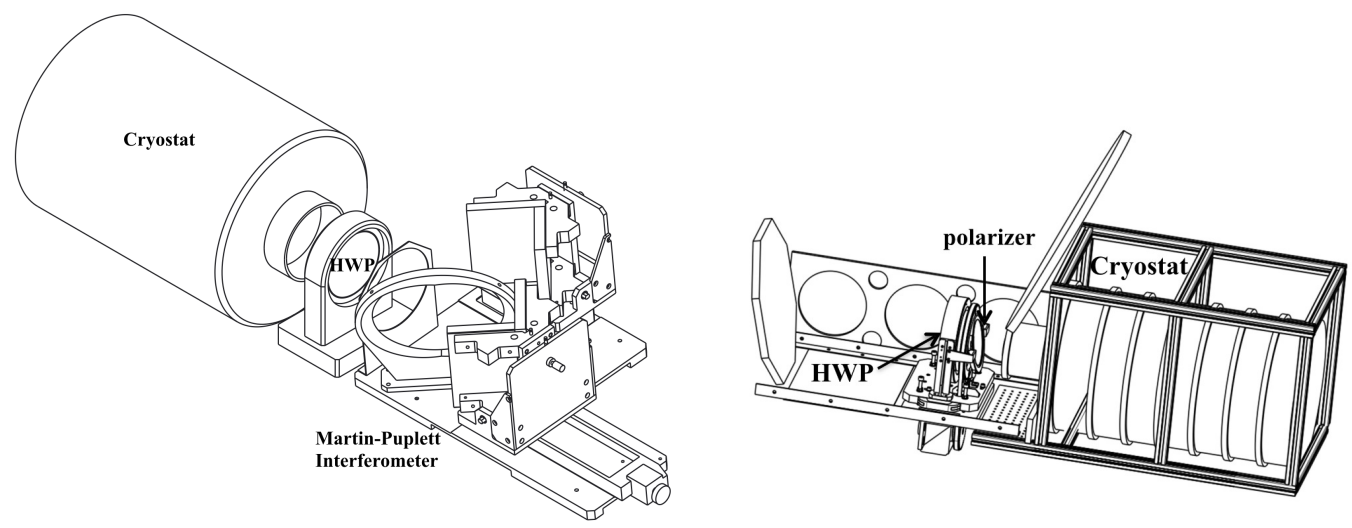

Fig. 1. Left: laboratory instrumental setup: the NIKA cryostat, the HWP in a fixed position, the polarizer mounted inside the cryostat and a MartinPuplett interferometer. Right: instrumental setup for polarization measurements at the telescope with the last two mirrors of the optics chain and the polarization module with the HWP and the stepper motor mounted in front of the entrance window of the cryostat. The polarizer is tilted by approximately $10 \mathrm{deg}$ with respect to the optical axis to avoid standing waves.

$M_{\mathrm{HWP}}=\frac{1}{2}\left(\begin{array}{lll}\alpha^{2}+\beta^{2} & \left(\alpha^{2}-\beta^{2}\right) \cos 2 \theta & \left(\alpha^{2}-\beta^{2}\right) \sin 2 \theta \\ \left(\alpha^{2}-\beta^{2}\right) \cos 2 \theta & \left(\alpha^{2}+\beta^{2}\right) \cos ^{2} 2 \theta+2 \alpha \beta \sin ^{2} 2 \theta \cos \phi & \left(\alpha^{2}+\beta^{2}-2 \alpha \beta \cos \phi\right) \cos 2 \theta \sin 2 \theta \\ \left(\alpha^{2}-\beta^{2}\right) \sin 2 \theta & \left(\alpha^{2}+\beta^{2}-2 \alpha \beta \cos \phi\right) \cos 2 \theta \sin 2 \theta & \left(\alpha^{2}+\beta^{2}\right) \sin ^{2} 2 \theta+2 \alpha \beta \cos ^{2} 2 \theta \cos \phi\end{array}\right)$.

anti-reflection coating was added to the HWP to maximize the transmission across the band. The HWP was mounted into a mechanical modulator actioned by a stepper motor synchronously controlled by the NIKA acquisition system. The power of the motor was chosen so that a typical stable rotation frequency of $5 \mathrm{~Hz}$ could be achieved during observational campaigns of at least one week.

The combined action of the continuously rotating HWP and the analyzer leads to a modulation of the input linear polarization at four times the mechanical rotation frequency, $\omega_{\mathrm{P}}=2 \pi v_{\mathrm{P}}$. Thus, setting aside calibration and system imperfections, each LEKID in the focal plane measures the following combination of the three Stokes parameters (commonly used to represent the time-averaged polarization state of electromagnetic radiation; for a review on polarization basics we refer to Collett 1992) $I$, $Q$, and $U$ :

$m=I+\rho_{\mathrm{pol}}\left\{Q \cos \left(2 \psi(t)+4 \omega_{\mathrm{P}} t\right)+U \sin \left(2 \psi(t)+4 \omega_{\mathrm{P}} t\right)\right\}$,

where $\psi(t)$ is the angle between the analyzer and the reference axis of the polarization reference frame, that is, the direction along which $U=0$ and $Q>0$, and $\rho_{\text {pol }}$ is the polarization efficiency of the full system.

\section{Laboratory characterization of the polarimetric module}

We begin this section by introducing the main parameters used in the characterization of the NIKA HWP. Following Savini et al. (2006), the Mueller matrix of a realistic HWP can be written as represented in Eq. (2).

$\alpha$ and $\beta$ represent the normalized transmission coefficients on the ordinary and extraordinary axes of the HWP. The HWP phase shift angle between the ordinary and extraordinary axes is noted as $\phi . \theta$ represents the angle of the HWP ordinary and extraordinary axes with respect to the polarization reference frame.

\section{See Eq. (2) above}

A first characterization of the properties of the NIKA HWP was carried out after fabrication at Cardiff University. In particular, the HWP phase shift angle $\phi$ was measured over the full bandwidth from 100 to $350 \mathrm{GHz}$, as shown on the top panel of Fig. 2. In the bottom panel of the figure, we show the spectral transmission of the NIKA HWP for different rotation angles; an attenuation of the signal is observed as expected. In order to estimate the performance of the whole NIKA polarization chain, we performed laboratory measurements of the system transmission. We used a polarizing Martin Puplett Fourier Transform Spectrometer (MPFTS) to characterize the spectral transmission of the system. The MPFTS produces the difference between the power of two input polarized beams that come from two black bodies at different temperatures (ambient ECCOSORB and warmed ECCOSORB) modulated by a rotating wire-grid polarizer. An array of LEKIDs was placed facing the MPFTS inside a NIKA-type dilution cryostat, which cools down the optics, the analyzer and the LEKIDs to a temperature of approximately $100 \mathrm{mK}$. The analyzer consists of a wire-grid and it is considered as ideal in the following. A schematic view of the instrumental setup is shown in the left panel of Fig. 1.

We performed a total of eight independent measurements by varying the angle of the NIKA HWP axis with respect to the optical axis. During each transmission measurement the HWP was kept fixed in a defined position. We achieved a spectral resolution of approximately $3.5 \mathrm{GHz}$, which corresponds to an approximately $44 \mathrm{~mm}$ excursion of the roof mirror of the MPFTS. We covered the bandwidth of interest by considering a total of 80 steps for transmission measurement for a total of 

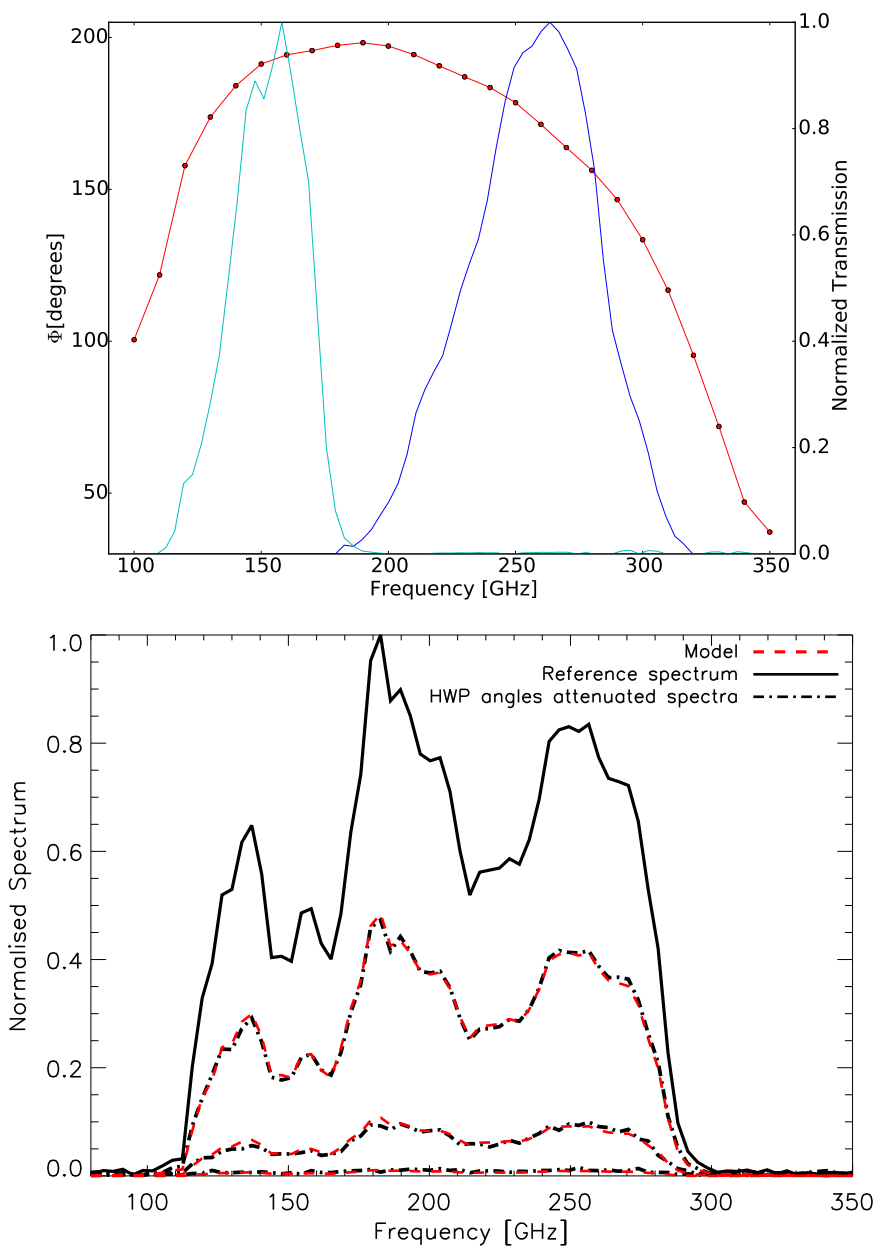

Fig. 2. Top: phase shift angle as a function of frequency for the NIKA HWP. The transmission for the two NIKA frequency bands at 1 (blue) and 2 (cyan) $\mathrm{mm}$ are also plotted for illustration. Bottom: spectral transmission of the NIKA HWP for different angles. The red curve corresponds to the best-fit model for the intermediate angle data. The maximum transmission corresponds to an angle of $46.8^{\circ}$ with respect to the HWP zero (optical axis). Attenuated spectra at $72^{\circ}, 79^{\circ}$, and $86.4^{\circ}$ (top to bottom curves) are also shown.

9 min integration time. As the MPFTS polarizer and the analyzer transmission axis were set perpendicularly to each other, prior to any measurement, we rotated the NIKA HWP to find a zero-point initial position, which maximized the measured LEKID signal. By rotating the HWP for each measurement we rotated the polarization of the MPFTS output signal. As the analyzer was kept at a fixed position, this induced an attenuation of the signal measured by the LEKIDs. This can be observed in the bottom panel of Fig. 2 where we present the measured transmission as a function of frequency for four HWP positions going from the maximum (black solid line) to the minimum of transmission (dashed solid line) spectra. We find that the angle between these two transmissions is $46.8 \pm 1.8^{\circ}$, which agrees with the expected $45 \mathrm{deg}$ from Eq. (2). A $1.8^{\circ}$ uncertainty is taken considering that the motor completes 100 steps per tour of the HWP. Therefore, it is the precision associated to the determination of the HWP zero, corresponding to its optical axis in the cabin reference frame.

We used previous transmission spectrum measurements to determine the $\alpha$ and $\beta$ transmission coefficients describing the
HWP. Taking the maximum transmission spectrum described above as a reference, we fitted for the other transmission spectra taken at different angles of the HWP with respect to the reference polarization axes. We assumed the HWP model in Eq. (2) and accounted for the analyzer facing the LEKID array. The phase shift angle, $\phi$, was set using the values per frequency measured at Cardiff University and presented above. Note that as we are using the maximum transmission spectrum as a template, we are not sensitive to an absolute attenuation amplitude. Therefore, to break the degeneracy between $\alpha$ and $\beta$, we fixed $\alpha$ to unity and only fitted for $\beta$. Using a least square minimization, we find $\beta=0.999 \pm 0.005$ at $1.15 \mathrm{~mm}$ and $\beta=0.924 \pm 0.005$ at $2.05 \mathrm{~mm}$. The best fit models obtained for each position of the HWP are plotted in red in Fig. 2.

We define the polarization efficiency from Eq. (2) as $\rho_{\text {pol }}=$ $(1-2 \gamma) / 2$, where $\gamma=\frac{\alpha \beta \cos (\phi)}{\alpha^{2}+\beta^{2}}$. Using the effective HWP phaseshift in the NIKA bands, we find $\rho_{\text {pol }}=0.9956 \pm 0.0002$ at $1.15 \mathrm{~mm}$ and $0.9941 \pm 0.0002$ at $2.05 \mathrm{~mm}$. These values are accounted for in the final absolute calibration of our polarization maps. Finally, as a consequence of the small difference observed between the $\alpha$ and $\beta$ transmission coefficients, we expect the modulation of the incoming intensity and polarization around the second harmonic of the HWP rotation (see Fig. 6). However, as presented in Sect. 4.4, we are not sensitive to this effect in the final maps as it is accounted for in the data processing.

\section{Reconstruction of the NIKA Stokes parameter maps}

We here describe the specificities of NIKA's polarization modulation strategy and data analysis. We start by giving more details on the fast and continuous rotation of the HWP and how it impacts the signal. We then present specific systematics associated to the rotating HWP and the optics, and how we handle them.

\subsection{Polarization modulation with a continuously rotating HWP}

The key aspect for reconstructing polarization with NIKA is the rotating HWP, which modulates the input polarization signal. Thus, we recall here the main lines guiding polarization modulation by a continously rotating HWP and its subsequent data analysis. For the sake of clarity, we consider the case of a polarized point source, which is observed under a classical raster scan strategy with constant declination while scanning along right ascension $\alpha$ at speed $\dot{\alpha}$. This type of scan being pseudoperiodical, the Fourier transform of the detector-observed timeordered data (time ordered information, hereafter TOI) shows peaks at harmonics of the scanning frequency (see Fig. 3), each peak containing the sum of the unpolarized and polarized fluxes of the source (see Eq. (1)). These peaks are damped by the instrument resolution at high frequency. The cut off at highspatial-resolution turns into a high-temporal-frequencies cutoff with typical Gaussian width $F W H M_{v}=\dot{\alpha} / 2 \pi F W H M$. This beam cutoff defines the signal band. According to Eq. (1), when we rotate a HWP in front of an analyzer, the polarized fraction of the signal is modulated at four times the mechanical rotation frequency of the HWP. Therefore, this shifts the polarized content of the signal at higher frequencies and recenters the signal band around the fourth harmonic of the mechanical rotation of the HWP (see Fig. 3 top). It is therefore clear that a low pass filter applied to the data above the beam cutoff and below the polarization signal band will preserve the unpolarized signal band 


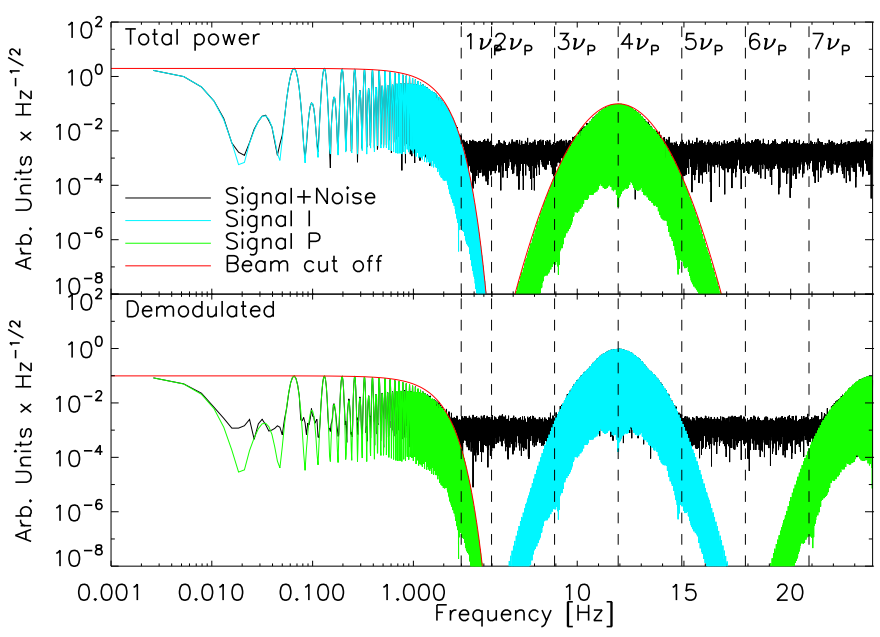

Fig. 3. Top panel: power spectrum of a simulated TOI for a polarized point source observed under a raster scan with a continuously rotating HWP facing a polarization analyzer. The raw signal plus noise TOI (black) has its total intensity content highlighted in cyan and the polarized content in green. The polarization signal band is centered on the fourth HWP harmonics while the intensity signal band lays at lower frequencies. Bottom panel: TOI after demodulation (see Sect. 4.1). We observe that half of its Stokes $Q$ content has been put at low frequency while the Stokes $I$ content and the remaining polarized contents are shifted at frequencies higher than the signal band.

while rejecting the polarized content and high-frequency noise. We refer to this type of TOI as "pure- $I$ " TOI in the following. To recover the polarization Stokes parameters $Q$ and $U$, we use a classic demodulation procedure discussed, for example, in Haykin (2008) and also adopted by Johnson et al. (2007). We build two reference signals:

$$
\begin{gathered}
r e f_{Q}=\cos \left(2 \psi(t)+4 \omega_{\mathrm{P}} t\right), \\
r e f_{U}=\sin \left(2 \psi(t)+4 \omega_{\mathrm{P}} t\right) .
\end{gathered}
$$

We multiply our TOIs by these reference signals and according to Eq. (1), obtain, for example $Q / 2+I \cos \left[2 \psi(t)+4 \omega_{\mathrm{p}} t\right]+\ldots Q$ and $U$ terms around the 8th harmonics of the HWP rotation. We have thus "demodulated" the $Q$ content of the TOI and brought it back to low frequencies, while rejecting the $U$ and $I$ content at high frequencies. A low pass filter above the beam cutoff and below the tail of the modulated intensity therefore provides a "pure- $Q$ " TOI (see the bottom panel of Fig. 3). Equivalently multiplying by $\sin \left[2 \psi(t)+4 \omega_{\mathrm{P}} t\right]$ and filtering we obtain a "pure- $U$ " TOI.

\subsection{Polarization measurements at the telescope}

The NIKA polarization setup at the telescope was similar to the one in the laboratory as shown on the right panel of Fig. 1. The HWP and analyzer were placed in the same mount facing the cryostat window in the optical axis of the Nasmyth cabin. Thus, the polarization reference frame was defined perpendicularly to the optical axis in Nasmyth coordinates.

During polarization observations the HWP was continuously rotated to modulate the input polarization signal as discussed in Sect. 4.1. The optimal HWP rotation speed is constrained by several factors including scanning speed and the scale of atmospheric variations. Usual scanning speeds for NIKA were approximately a few tens of arcsecs/s. Atmospheric turbulence and variations across the scan transform into $1 / f$-like noise with typical knee frequency below $1-2 \mathrm{~Hz}$ for reasonable atmospheric conditions, as illustrated on Fig. 4 that shows measured raw TOI (black curve) for a single detector as a function of sample number (left) and its corresponding power spectrum (right). In addition to the atmospheric noise, a subdominant detector correlated-noise component had been found in the NIKA data and identified as electronic-based noise.

Rotating the HWP such that four times its rotation speed places the polarization signal well above $2 \mathrm{~Hz}$ permits a natural rejection of these two major noise components. If the rotation is also fast compared to the scanning speed and the angular resolution, the three Stokes parameters can be derived quasisimultaneously, thus rejecting further residual low-frequency drifts. Finally, a fast rotation places possible parasitic signals at harmonics of the rotation frequency outside the signal band (see Sect. 4.3 for more details). Both the polarization modulation and parasitic signals can be clearly observed in Fig. 4 as high-frequency peaks in the TOI spectrum at harmonics of the HWP rotation frequency. A fast rotation sets tight mechanical constraints on the stepper motor and imposes a faster data acquisition with respect to unpolarized observations. That is why we chose, as discussed above, to acquire data at $47.7 \mathrm{~Hz}$, rotate the HWP at $2.98 \mathrm{~Hz}$, and to limit our scanning speed to 26 arcsecs/s. This provides five measurements of $I, Q$, and $U$ per FWHM, well within the Nyquist limit, even at a LEKID timeline level. Each of these five measurements results from the combination of four data samples taken at four different HWP angles. To conclude, we are then able to reconstruct the Stokes parameters per detector, with high spatial and temporal redundancy and to reject atmospheric noise from the polarization signal band.

\subsection{Systematic HWP synchronous signal correction}

Imperfections of the HWP modulate the background and lead to a strong additional parasitic signal, highly peaked at harmonics of the HWP rotation frequency $v_{\mathrm{P}}$ as shown in the right panel of Fig. 4. Such a HWP synchronous signal (HWPSS) was previously observed by Maxima (Johnson et al. 2007) and EBEX (Chapman et al. 2014), although the HWP driving mechanisms were different. Like them, we find that the signal is well fitted by a sum of harmonics of the HWP rotation frequency, $v_{\mathrm{P}}$ with amplitudes slowly and linearly drifting. This is illustrated in Fig. 5 where we plot the time variation of the cosine $A_{n}$ (red) and sine $B_{n}$ (blue) component amplitudes of the first four harmonics of the HWP rotation frequency along a scan of Uranus. To derive these amplitudes, we use a simplified model of Eq. (4) to fit only the constant part of amplitudes $A_{n}$ and $B_{n}$ over chunks of $30 \mathrm{~s}$. We observe that the relative variation is at most $2 \mathrm{mJy} / \mathrm{s}$, mainly dependent on the background (Stokes $I$ ). We therefore model this additional parasitic signal as a Fourier series of the harmonics of the HWP rotation frequency

$$
\begin{aligned}
\operatorname{HWPSS}(t)= & \sum_{n=1}^{8}\left(A_{n}^{0}+\epsilon_{A_{n}} t\right) \cos n \omega_{\mathrm{P}} t \\
& +\left(B_{n}^{0}+\epsilon_{B_{n}} t\right) \sin n \omega_{\mathrm{P}} t .
\end{aligned}
$$

We consider up to eight harmonics of $v_{\mathrm{P}}$ and explicitly include a linear variation of the amplitude coefficients both for the sine and cosine components when we fit this model on data. We perform a simple linear fit of the previous model to the full calibrated and opacity corrected (see Sect. 4.5 for details) raw TOI to derive the best-fit amplitude coefficients. With these coefficients in hand, we construct a template of the HWPSS, which is then subtracted from the raw calibrated TOI. An illustration of this procedure is shown in the left panel of Fig. 4 where the 

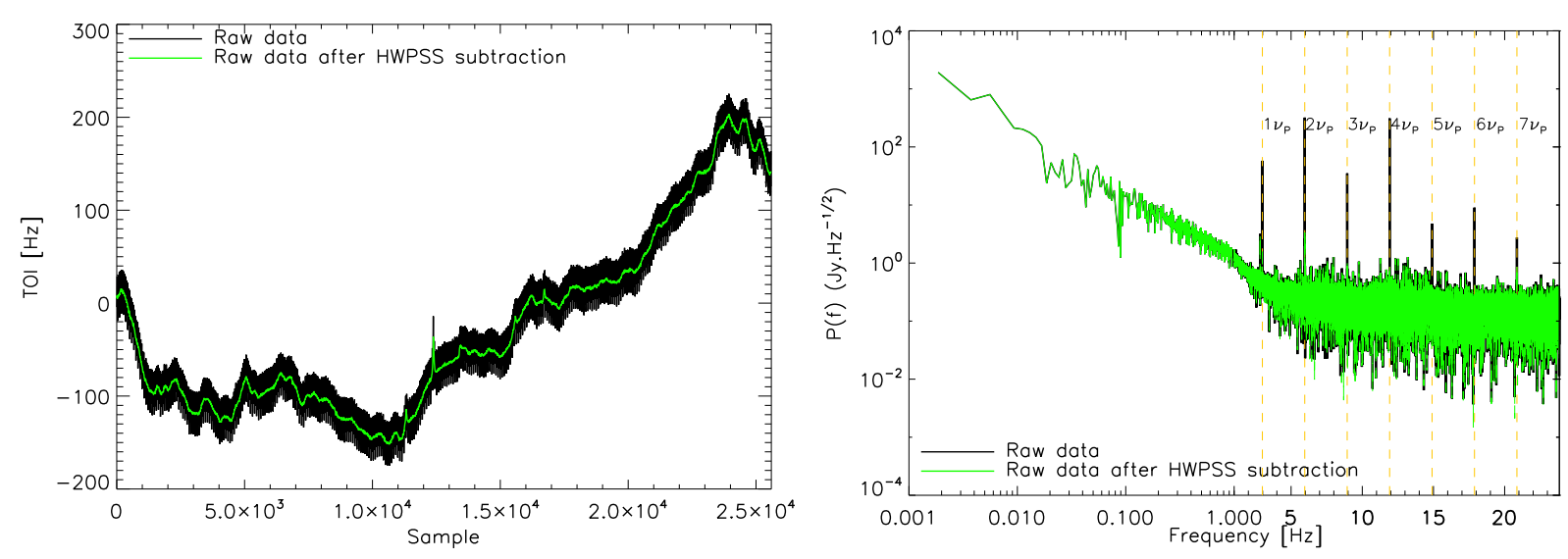

Fig. 4. TOI (left) and power spectrum (right) of an observation of Orion OMC-1 for a single KID. Raw data are presented in black and the Half Wave Plate systematic signal (HWPSS) subtracted data in green.

raw TOI is shown before (black) and after (green) subtraction of the HWPSS template. Equivalently, the right panel of the figure presents the power spectrum of the raw TOI before (black) and after (green) the subtraction of the HWPSS template. Thanks to this procedure, the HWPSS residuals are reduced to the noise level.

\subsection{NIKA demodulation procedure and map making}

After subtraction of the HWPSS, we use the lock-in procedure described in Sect. 4.1 to separate the raw TOI per KID into pure Stokes $I, Q$, and $U$ TOIs. These TOIs are decorrelated from a common mode to remove the residual atmospheric and electronic noise, and then projected into Stokes $I, Q$, and $U$ maps. We use the same decorrelation procedures developed for the intensity-only NIKA observations (see Adam et al. 2014; Catalano et al. 2014, for details). In particular we use a common mode decorrelation, masking the source and using only the pixels outside the source to decorrelate. A low pass filter is also applied to the pure Stokes $I, Q$, and $U$ TOIs to reject highfrequency noise. The frequency cutoff is set slightly below the HWP rotation frequency. For the projection we use an inverse noise-weighting procedure and account for instrumental flags indicating unreliable data samples or detectors (see Adam et al. 2014; Catalano et al. 2014, for details). In the case of the $Q$ and $U$ maps, this map-making procedure is almost optimal map making because the noise is expected to be nearly white in the pure Stokes $Q$ and $U$ TOIs. This is illustrated in Fig. 6 where we present, in black, the power spectra of the pure Stokes $I, Q$, and $U$ TOIs obtained by applying the lock-in procedure to the raw TOI of Fig. 4. We observe that the power spectra of the pure Stokes $Q$ and $U$ TOIs are almost flat indicating, as expected, a significant, although not complete, reduction of the contribution from atmospheric fluctuations that shows a $1 / f$-like component on a pure $I$ TOI. The best-fit power spectrum models are presented in red in Fig. 6. Using simulations, we have proven that the residual $1 / f$-like component in $Q$ and $U$ is consistent with residual atmospheric emission induced by intensity to polarization leakage as discussed in Sect. 4.6. We also show the power spectra, after applying the decorrelation procedure, in cyan. This leads to a significant reduction of the $1 / f$-like noise in the pure Stokes $I$ TOI power spectrum and of the residual low-frequency tail on the pure Stokes $Q$ and $U$ TOIs.
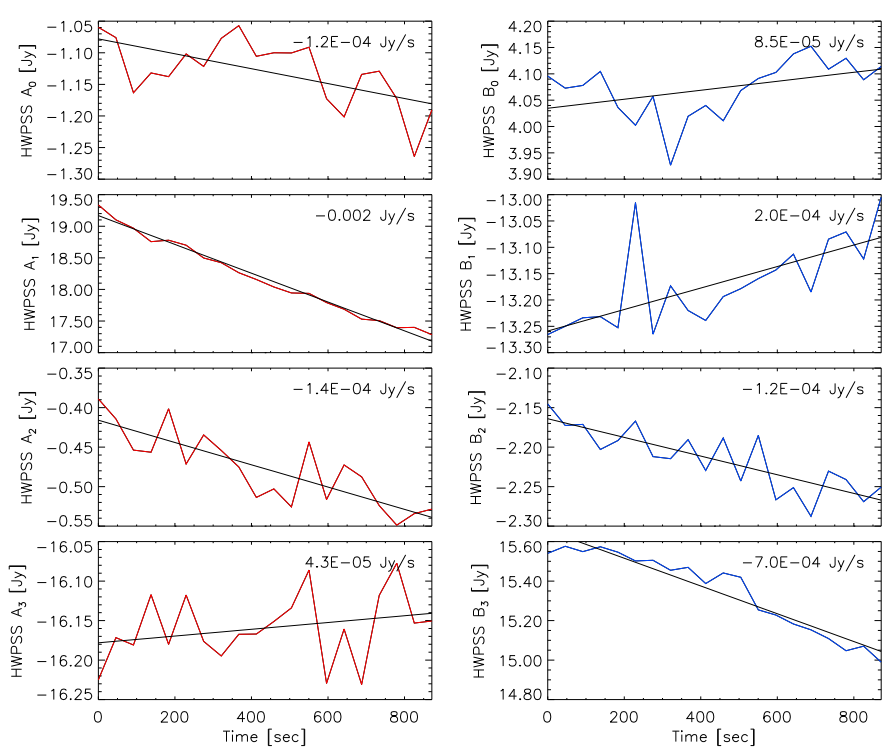

Fig. 5. Absolute amplitudes of the four main harmonics of the HWPSS as a function of time. Each point is a measurement of these amplitudes on a chunk of approximately $30 \mathrm{~s}$. The amplitudes show a slow and linear drift in time.

\subsection{Absolute calibration and inter-calibration}

Absolute calibration is performed in the same way as for the intensity-only NIKA observations (Adam et al. 2014; Catalano et al. 2014). We use Uranus as our main absolute flux calibrator and compute calibration factors per KID by fitting a 2D Gaussian to the data. We take the measured FWHMs of 12 arcsec at $1.15 \mathrm{~mm}$ and 18.2 arcsec at $2.05 \mathrm{~mm}$. For the data presented in this paper, we have $14 \%$ uncertainty on absolute calibration at $1.15 \mathrm{~mm}$ and $5 \%$ at $2.05 \mathrm{~mm}$. The standard deviation of the flux distribution on Uranus directly gives the calibration error associated to the estimation of point source flux values. After calibration, the data are given in units of Jy/beam. We also correct the raw data from atmospheric absorption using the NIKA instrument as a taumeter following the procedure described in Catalano et al. (2014).

\subsection{Instrumental polarization and leakage}

Like most authors, we define instrumental polarization as the ability of the instrument to convert incident unpolarized total 

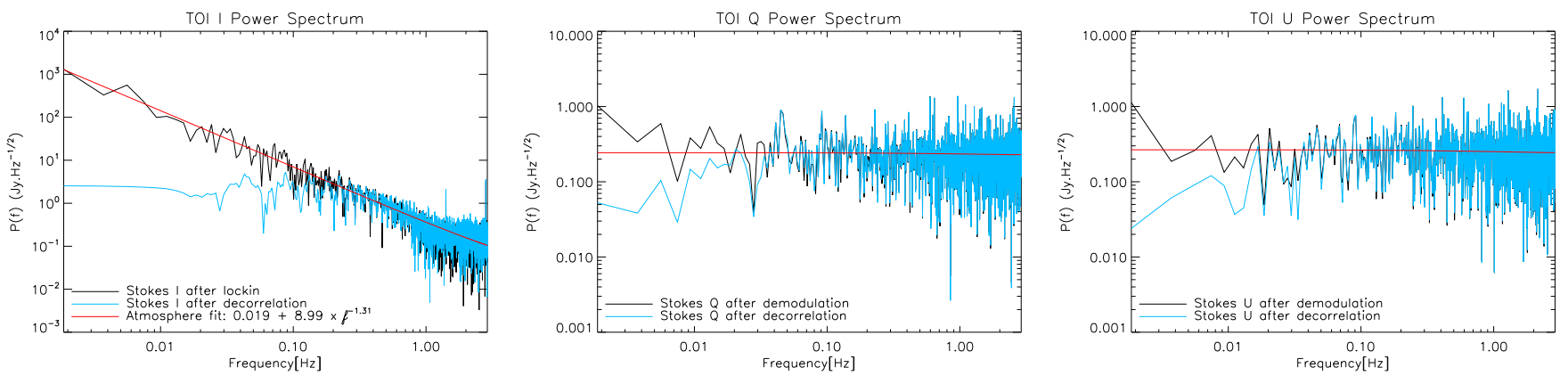

Fig. 6. From left to right: power spectra of the Stokes $I, Q$, and $U$ pure TOIs (black) after applying the lock-in procedure to the raw data in Fig. 4 . A bandpass filter $([0.01,2.9] \mathrm{Hz})$ was applied to reject any high-frequency noise and half wave plate systematic signal (HWPSS) residual at the fourth harmonics. In blue we also show the power spectra after applying the decorrelation procedure to the demodulated data. See Sect. 4.4 for details.

power into a polarized signal. This conversion can then take various forms, as we describe in this section. To estimate the level of instrumental polarization at the telescope we repeatedly observed Uranus, which is assumed to be unpolarized, (Wiesemeyer et al. 2014 find a polarization of $\sim 0.1 \%$ ), and bright (47.2 Jy at $1.15 \mathrm{~mm}, 16.4 \mathrm{Jy}$ at $2.05 \mathrm{~mm}$ ). It has an apparent diameter at the time of observations of 3 arcsec and can therefore be approximated as a point source compared to our beams. Figure 7 shows Stokes $I, Q$, and $U$ maps of Uranus in Nasmyth (i.e., cabin) coordinates at 1.15 and $2.05 \mathrm{~mm}$ in panels $a$ and $b$, respectively. For each panel, the top row shows the raw NIKA maps after projection of the decorrelated pure Stokes $I, Q$, and $U$ TOIs. We observe a significant signal in $Q$ and $U$ that indicates a non-zero level of instrumental polarization. We mainly identify a bipolar pattern, partially consistent between the two bands, with a peak to peak amplitude at the level of $3 \%$ of the total intensity peak. Such an effect has already been observed in other experiments (e.g. Thum et al. 2008 and BICEP2 and Keck Array Collaborations et al. 2015). We performed a large number of observations of Uranus at different elevation angles. From these observations we concluded that the observed leakage effect is fixed in Nasmyth coordinates. Although we still lack a convincing physical interpretation of the observed signal, we can model it as leakage from total intensity $I$ into $Q$ and $U$, and write the observed Stokes parameters in Nasmyth coordinates as

$$
\begin{aligned}
\hat{I}_{N} & =B_{I} * I_{N}+N_{I}, \\
\hat{Q}_{N} & =B_{I} * Q_{N}+\mathcal{L}_{N}^{I Q} * I_{N}+N_{Q}, \\
\hat{U}_{N} & =B_{I} * U_{N}+\mathcal{L}_{N}^{I U} * I_{N}+N_{U} ;
\end{aligned}
$$

where $I_{N}, Q_{N}$, and $U_{N}$ are the original sky Stokes parameters in Nasmyth coordinates. $B_{I}$ represents the NIKA response pattern and $*$ denotes spatial convolution. The different noise contributions discussed above are accounted for in $N_{I, Q, U}$. Finally, we model the leakage term as the convolution of the original intensity map with response-pattern-like kernels $\mathcal{L}_{N}^{I Q}$ and $\mathcal{L}_{N}^{I U}$ for $Q$ and $U$, respectively. These two kernels are directly estimated from the $Q_{N}$ and $U_{N}$ maps of Uranus presented in Fig. 7, which, as discussed above, can be considered as a point source. Note that here we assume no modification of the intensity signal and we account for any loss of power at the calibration stage.

One way to correct for this instrumental polarization is to convolve the observed polarization maps (Eqs. (6) and (7)) by the main instrumental gaussian beam $B_{I}$ and subtract the convolution of the observed intensity map $\hat{I}_{N}$ (Eq. (5)) by the leakage kernels. The results are polarization maps that are free from instrumental polarization leakage, but with a degraded resolution at 17 and 25.5 arcsec at 1.15 and $2.05 \mathrm{~mm}$ respectively, and with an extra fraction of the total intensity noise convolved by the leakage kernels. To avoid these two artefacts, we have devised a dedicated algorithm:

1. With the demodulation and projection techniques presented in Sect. 4, we build maps of Stokes $I, Q$, and $U$ of the observed signal in equatorial coordinates. These maps can be the result of multiple observation scans to obtain the best possible signal to noise. We only need the $I$ map in the following to derive the leakage signal that we want to subtract.

2. We rotate the $I$ map into Nasmyth coordinates to obtain $\hat{I}_{N}$ for a given scan. The needed rotation angle, which is the combination of the elevation and the parallactic angles, varies along the scan. However, we find that not accounting for this variation during a given scan leads to negligible differences.

3. Build Fourier space convolution/deconvolution kernels of the form $\mathcal{L}_{I Q} / B_{I}$ and $\mathcal{L}_{I U} / B_{I}$ from observations of Uranus.

4. Multiply the Fourier transform of $I^{N}$ by the above kernels and transform the result back into real space to build maps of leakage from $I$ into $Q$ and $U$.

5. Deproject the obtained maps with the actual scanning strategy to produce $Q$ and $U$ TOIs that are then subtracted from the decorrelated pure Stokes $Q$ and $U$ TOIs presented in Sect. 4.4.

6. Project these corrected TOIs onto final maps following the same map-making procedure as in Sect. 4.4.

A similar technique developed to reduce the leakage effect observed with XPOL instrument from the IRAM $30 \mathrm{~m}$ telescope is presented in Hezareh et al. (2013). In Fig. 7, the bottom rows of panels $\mathrm{a}$ and $\mathrm{b}$ show the final Nasmyth-coordinate-UranusStokes $I, Q$, and $U$ maps after leakage correction using the above algorithm. Note that to compute the leakage kernels we use a set of independent Uranus observations to cross check the efficiency of the procedure. We observe that after leakage correction the residual leakage in the $Q$ and $U$ maps of Uranus drops below $1 \%$. We now see a residual signal that we interpret as "straightforward" instrumental polarization, that is, an induced polarization directly proportional to $I$. This instrumental polarization is below $1 \%$ for both $Q$ and $U$, and is removed by subtracting the relative fraction of the total intensity map from our polarization maps.

Applying this data-reduction algorithm to observations of sources with polarized emission allows us to correct for leakage effects in this context as well. The top row of Fig. 9 shows 
(a) $1.15 \mathrm{~mm}$ raw (top row) and leakage corrected (bottom row) Stokes $I, Q$ and $U$ maps.
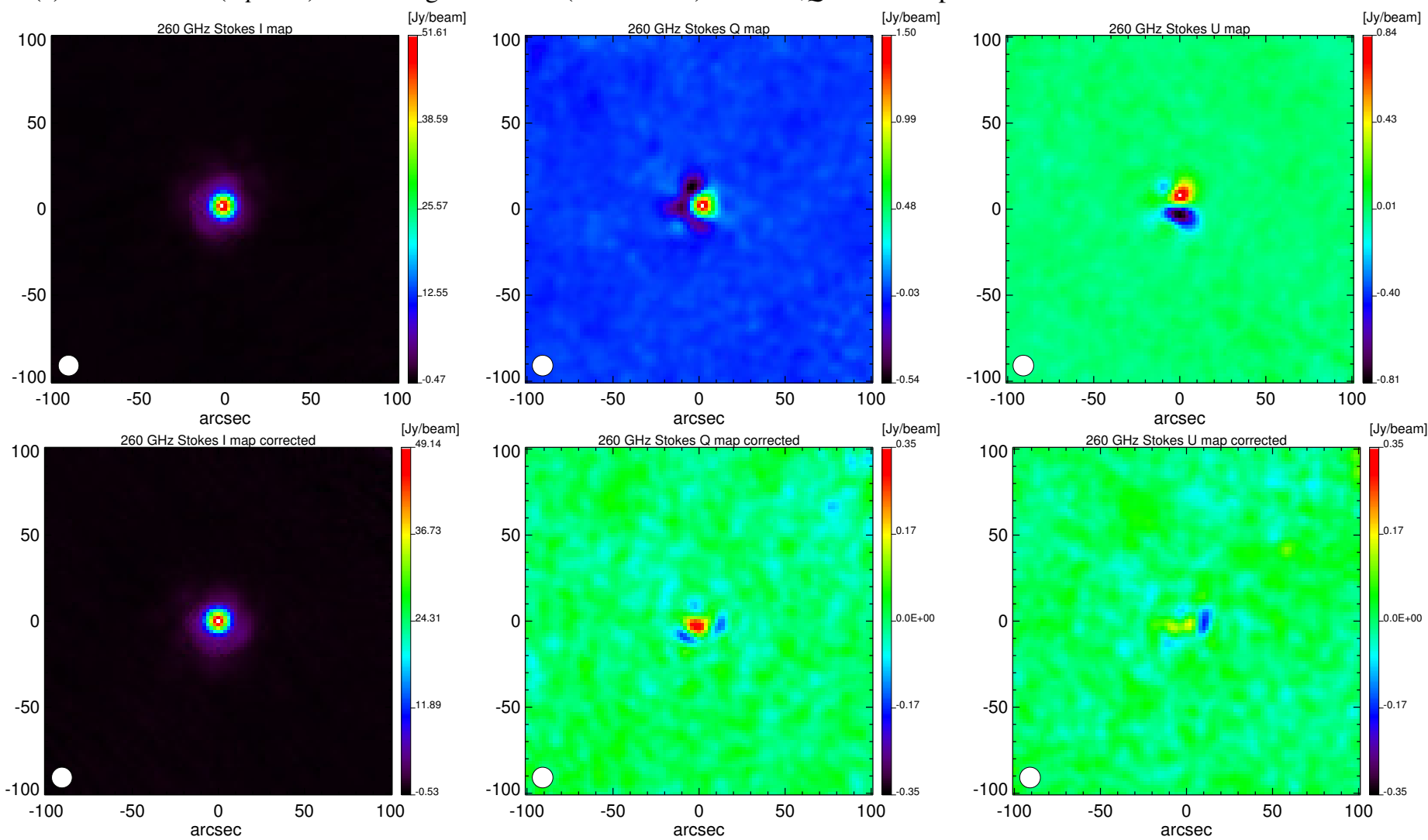

(b) $2.05 \mathrm{~mm}$ raw (top row) and leakage corrected (bottom row) Stokes $I, Q$ and $U$ maps.
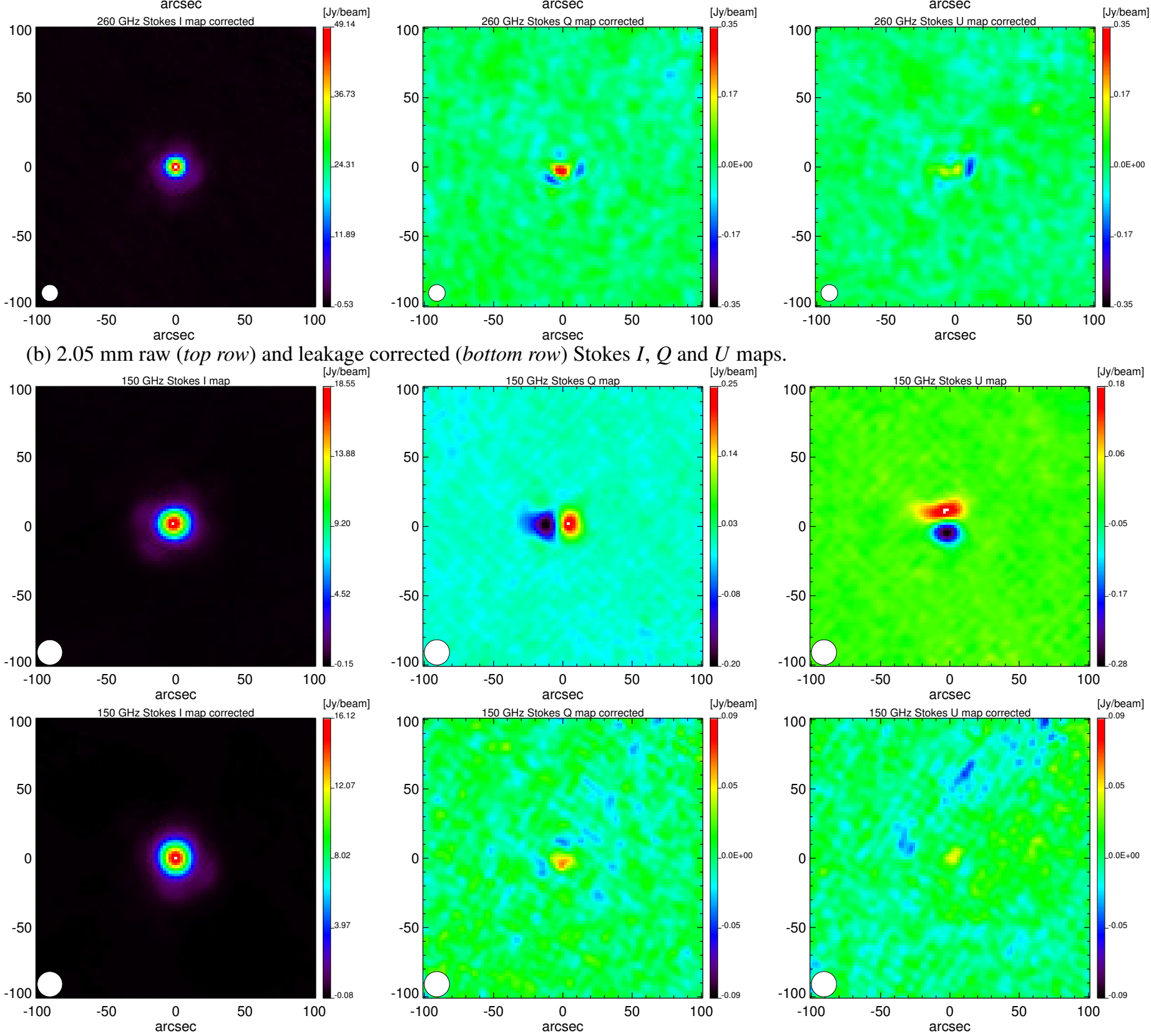

Fig. 7. Uranus Stokes $I, Q$ and $U$ maps in Nasmyth coordinates at $260 \mathrm{GHz}$ a) and $150 \mathrm{GHz}$ b) before and after leakage correction. After the leakage correction, we are left with a residual instrumental polarization below $1 \%(0.7 \%$ at $1.15 \mathrm{~mm}$ and $0.6 \%$ at $2.05 \mathrm{~mm})$. 


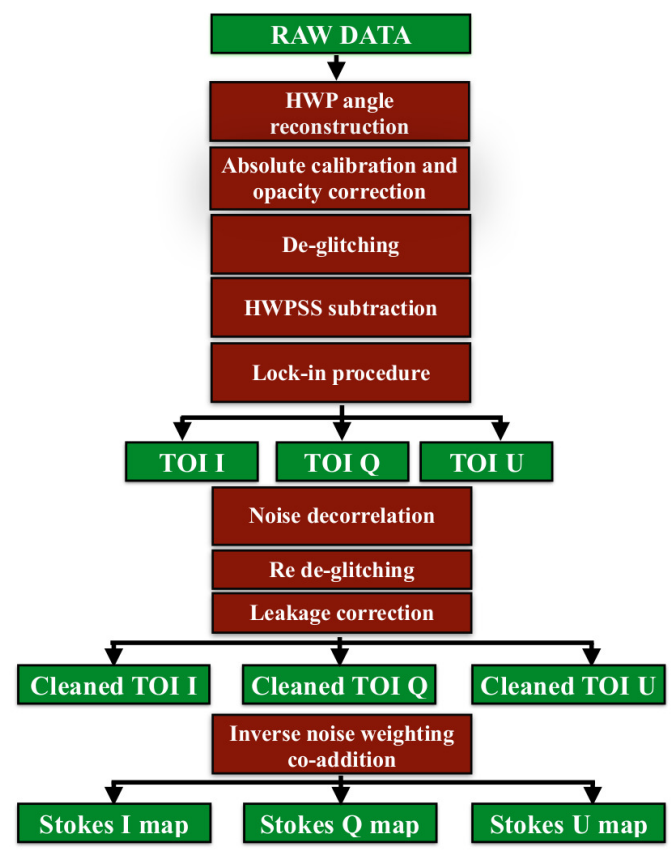

Fig. 8. Schematic view of the main procedures of the data processing pipeline from raw data to sky maps.

the NIKA Stokes $I, Q$, and $U$ maps at $2.05 \mathrm{~mm}$ of the quasar 3C 273 before leakage correction. We clearly see in the $Q$ and $U$ maps a bipolar structure similar to the one observed on the Uranus maps. The bottom row of the figure presents the leakagecorrected maps, which show no residual bipolar structure but slightly increased noise contribution. Indeed, the division by $\tilde{B}_{I}$ boosts the signal on small angular scales and therefore the noise, but the damping of $\mathcal{L}_{I Q}$ compensates and ensures regularization. In the case of point-source observations, we can make this noise increase even smaller by simulating a point source with the measured flux and convolve it directly by $\mathcal{L}_{I Q}$ and $\mathcal{L}_{I U}$ to derive the leakage corrections. The noise in total intensity is then not involved in the process.

\subsection{Summary of data processing pipeline}

Figure 8 presents a schematic view of the main procedures used to convert the raw NIKA data into leakage corrected Stokes $I, Q$, and $U$ maps. The main steps are

1. Read raw data.

2. Reconstruct the position of the HWP and the corresponding angle with respect to the zero reference.

3. Compute absolute calibration and correct for atmospheric absorption.

4. Apply a basic de-glitching algorithm to remove spikes on the raw NIKA TOIs.

5. Reconstruct and subtract the HWPSS.

6. Apply lock-in procedure to the raw NIKA TOIs to build pure Stokes $I, Q$, and $U$ TOIs.

7. Apply the decorrelation procedure to the pure Stokes $I, Q$, and $U$ TOIs.

8. Apply a basic de-glitching algorithm to remove spikes on the pure Stokes $I, Q$, and $U$ TOIs.

9. Apply the leakage-correction algorithm to the decorrelated pure Stokes $I, Q$, and $U$ TOIs.
10. Apply the map-making procedure to the decorrelated and leakage-corrected pure Stokes $I, Q$ and $U$ TOIs.

11. Project the cleaned TOIs into maps.

\section{Validation of the quality of the NIKA polarization reconstruction on quasars}

\subsection{Quasar selection and previous observations}

During the NIKA February 2015 technical campaign we observed a selection of quasars to validate the quality of the reconstruction of the polarization signal with the NIKA camera. We have considered both bright and highly polarized quasars. As shown in Table 1, the selected targets were 3C 279, 3C 273, $3 \mathrm{C} 286$, and $0923+392$. We list in the following the main physical characteristics and polarization properties of the most commonly observed quasars at mm wavelength: 3C 286, 3C 279 and $3 \mathrm{C} 273$.

\section{$3 C 286$}

3C 286 is a compact steep-spectrum quasar at redshift $z=0.846$. The stability of this quasar in intensity and polarization in a large frequency range and the slow wavelength dependence make it a primary calibrator for polarization measurements. At centimeter wavelengths, where 3C 286 is commonly used as a primary polarization calibrator, observations show that the 3C 286 polarization angle (PA) has been stable for decades (Perley \& Butler 2013). At millimeter wavelengths the XPOL polarimeter (Thum et al. 2008) has monitored 3C 286 from 2006 to 2012 (Agudo et al. 2012). As presented in Table 2, XPOL observations show that 3C 286 is highly polarized, up to $14 \%$, with PA increasing slowly with frequency. These results have been confirmed by observations at $1.3 \mathrm{~mm}$ with CARMA (Hull \& Plambeck 2015) in May 2015 (see Table 2).

\section{$3 \mathrm{C} 279$}

The blazar 3C 279 is one of the brightest and best monitored flatspectrum quasars. It was the first object to exhibit apparent superluminal motion. The source of its strong radio to $\gamma$-ray emission is a relativistic jet of material ejected from the black hole in its centre (Wagner et al. 2015). 3C 279 is a variable source but strongly polarized up to $11 \%$. In Table 2 , we present results in terms of degree of polarization and PA from recent observations of 3C 279 by the SHARP polarimeter (Lee et al. 2015). These observations were performed in March 2014 at $350 \mu \mathrm{m}$. Simultaneous observations have been performed with the very long baseline interferometer (VLBI; Lee et al. 2014) at 3.5, 7, and $13 \mathrm{~mm}$.

\section{$3 \mathrm{C} 273$}

$3 \mathrm{C} 273$, the first quasar ever to be identified, is located in the constellation of Virgo at a redshift $z=0.158$ (Madsen et al. 2015). 3C 273 is the brightest and hence one of the best monitored active galactic nuclei (AGN). From radio to millimeter wavelengths, flares from the relativistic jet dominate the variability of 3C 273 (Abdo et al. 2010). 3C 273 shows relatively low polarization, approximately 3-4\% (see Table 2), at mm wavelengths. 

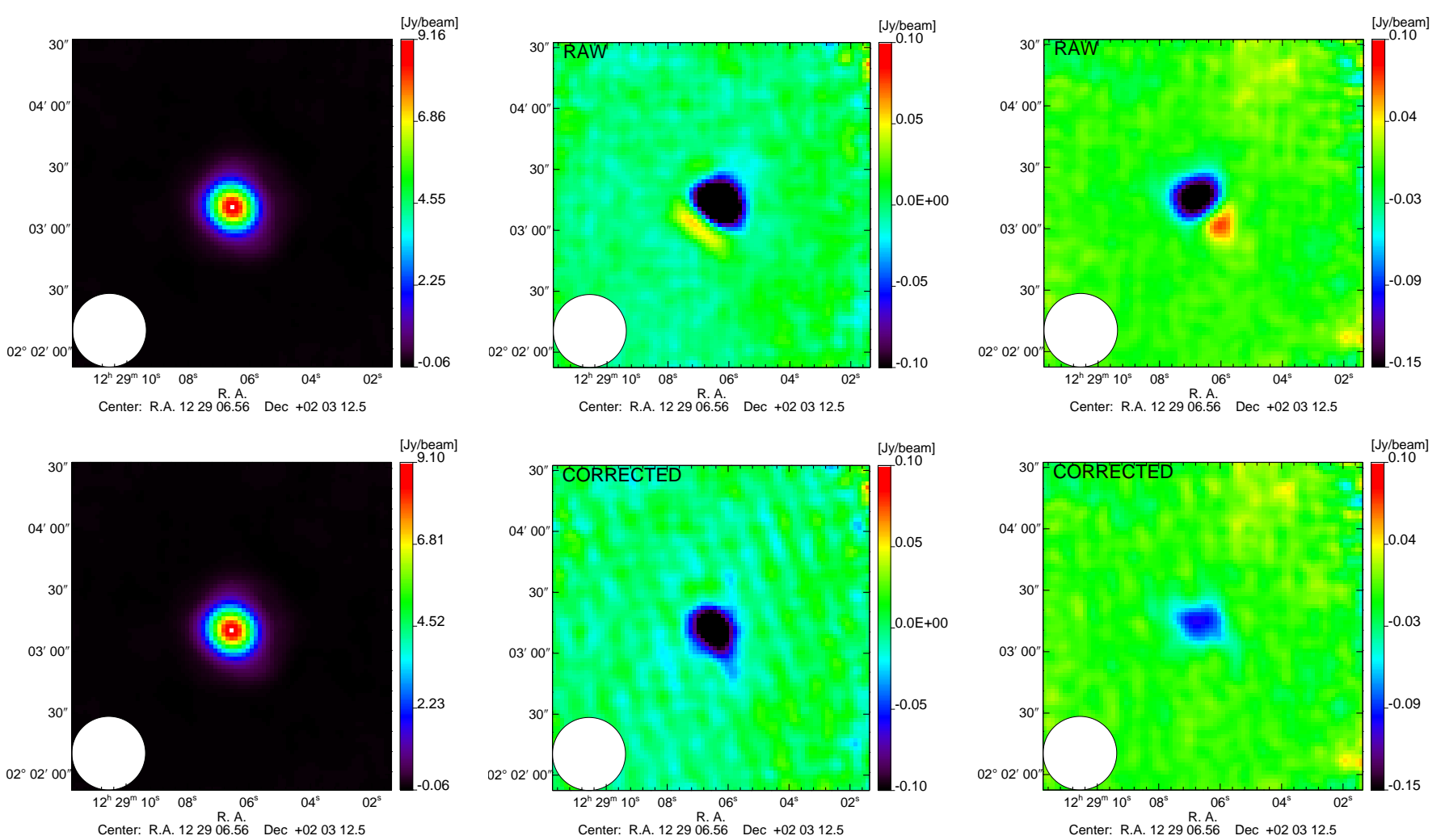

Fig. 9. I, $Q$, and $U$ maps of the quasar 3C 273 observed at $150 \mathrm{GHz}$ before (top) and after (bottom) correction for the leakage effect. The polarization angle and degree are reported in Table 1.

Table 1. NIKA measured intensity and polarization fluxes, polarization degree and angle at $260 \mathrm{GHz}$ and at $150 \mathrm{GHz}$ for the quasars observed during the February 2015 campaign.

\begin{tabular}{ccccccc}
\hline \hline Source & $\begin{array}{c}\text { Frequency } \\
\text { [GHz] }\end{array}$ & $\begin{array}{c}I \text { flux } \\
\text { [Jy] }\end{array}$ & $\begin{array}{c}Q \text { flux } \\
{[\mathrm{Jy}]}\end{array}$ & $\begin{array}{c}U \text { flux } \\
{[\mathrm{Jy}]}\end{array}$ & $\begin{array}{c}p \\
{[\%]}\end{array}$ & $\begin{array}{c}\psi \\
{\left[{ }^{\circ}\right]}\end{array}$ \\
\hline $3 \mathrm{C} 279$ & 260 & $8.52 \pm 0.28$ & $0.26 \pm 0.01$ & $0.79 \pm 0.03$ & $9.8 \pm 0.4$ & $35.9 \pm 0.5$ (stat) \pm 1.8 (syst) \\
& 150 & $12.21 \pm 0.58$ & $0.51 \pm 0.02$ & $1.04 \pm 0.05$ & $9.5 \pm 0.6$ & $31.9 \pm 0.7$ (stat) \pm 1.8 (syst) \\
\hline $3 \mathrm{C} 273$ & 260 & $6.35 \pm 0.22$ & $-0.22 \pm 0.01$ & $-0.01 \pm 0.01$ & $3.4 \pm 0.3$ & $-88.7 \pm 0.9$ (stat) \pm 1.8 (syst) \\
& 150 & $9.95 \pm 0.48$ & $-0.17 \pm 0.01$ & $-0.11 \pm 0.01$ & $2.0 \pm 0.1$ & $-74.1 \pm 1.1$ (stat) \pm 1.8 (syst) \\
\hline $3 \mathrm{C} 286$ & 260 & $0.27 \pm 0.01$ & $0.021 \pm 0.003$ & $0.033 \pm 0.004$ & $14.3 \pm 1.7$ & $30.0 \pm 2.5$ (stat) \pm 1.8 (syst) \\
& 150 & $0.51 \pm 0.03$ & $0.039 \pm 0.002$ & $0.056 \pm 0.002$ & $13.6 \pm 0.8$ & $28.0 \pm 0.9$ (stat) \pm 1.8 (syst) \\
\hline $0923+392$ & 260 & $2.04 \pm 0.06$ & $-0.002 \pm 0.005$ & $-0.066 \pm 0.005$ & $3.2 \pm 0.3$ & $-46.1 \pm 2.4$ (stat) \pm 1.8 (syst) \\
& 150 & $3.24 \pm 0.14$ & $-0.016 \pm 0.005$ & $-0.087 \pm 0.006$ & $2.7 \pm 0.2$ & $-50.4 \pm 1.7$ (stat) \pm 1.8 (syst) \\
\hline
\end{tabular}

\subsection{Polarization reconstruction accuracy}

Table 1 presents the Stokes $I, Q$, and $U$ fluxes measured by NIKA at 1.15 and $2.05 \mathrm{~mm}$ for the selected quasars. The fluxes have been measured using a simple aperture photometry procedure. The reported uncertainties account for inhomogeneities as well as for correlated noise in the maps (see Adam et al. 2016, for details).

In observations of linear polarization, it is common to represent the polarized signal in terms of polarization degree, $p$, and angle, $\psi$ :

$$
\begin{aligned}
& p=\frac{\sqrt{Q^{2}+U^{2}}}{I}, \\
& \psi=\frac{1}{2} \arctan \frac{U}{Q} .
\end{aligned}
$$

These definitions are not linear in $I, Q$, and $U$ and their naive estimation is biased by the noise. A non-Gaussian behavior is expected for $p$ and $\psi$ leading to both biases and wrong estimates of the uncertainties of $p$ and $\psi$. In particular regions of low polarized signal-to-noise ratio, when $Q \simeq U \simeq 0$, the noise measured on $Q$ and $U$ maps will yield a non-zero degree of polarization estimation. Ways to correct the bias in the estimation of $p$ have been proposed by Simmons et al. (1980) and more recently by Simmons \& Stewart (1985) and Montier et al. (2015) to whom we refer here. Although we have implemented the full likelihood based estimators of $p$ and $\psi$ according to the "1Dmarginal distributions" of Montier et al. (2015), in this first paper on test data, we focus on high-S/N results (especially on $I$ ) and are therefore in the limit where the estimator of the degree of polarization approaches:

$\hat{p} \simeq \sqrt{Q^{2}+U^{2}-\sigma_{q}^{2}-\sigma_{u}^{2}} / I$. 
A. Ritacco et al.: NIKA polarization performance

Table 2. Degree and angle of polarization of the NIKA observed quasars as measured by other experiments.

\begin{tabular}{ccccccc}
\hline \hline Source & Experiment & $\begin{array}{c}\text { Frequency } \\
{[\mathrm{GHz}]}\end{array}$ & $\begin{array}{c}p \\
{[\%]}\end{array}$ & $\begin{array}{c}\psi \\
{\left[{ }^{\circ}\right]}\end{array}$ & Observation date & Comments \\
\hline 3C 279 & SHARP and VLBI & $(857,85.7,42.8,23)$ & $10 \%-12 \%$ & $32-41$ & 2014, March & (Lee et al. 2015) \\
& XPOL & 260 & $11.79 \pm 0.29$ & $45.6 \pm 0.7$ & 2016, January & NIKA - XPOL joint session \\
\hline \multirow{2}{*}{$3 \mathrm{C}$ 273 } & XPOL & 86 & $1.1 \pm 0.0$ & $-37.8 \pm 0.9$ & 2015, February & NIKA - XPOL joint session \\
& XPOL & 229 & $3.6 \pm 0.2$ & $-76.8 \pm 1.6$ & 2015, February & NIKA - XPOL joint session \\
& XPOL & 260 & $1.59 \pm 0.18$ & $-71.8 \pm 3.1$ & 2016, January & NIKA - XPOL joint session \\
\hline \multirow{2}{*}{$3 \mathrm{C}$ 286 } & XPOL & 86 & $13.5 \pm 0.3$ & $37.3 \pm 0.8$ & 2006-2012 & (Agudo et al. 2012) \\
& XPOL & 229 & $14.4 \pm 1.8$ & $33.1 \pm 5.7$ & 2006-2012 & (Agudo et al. 2012) \\
& CARMA & 230 & & $39.1 \pm 1$ & 2015, May & (Hull \& Plambeck 2015) \\
& SMA & 230 & $11.5 \pm 2.5$ & $35.6 \pm 5.9$ & 2006 & (Marrone 2006) \\
& SMA & 340 & $15.7 \pm 0.8$ & $37.4 \pm 1.5$ & 2016 & (Hull et al. 2016) \\
& ALMA & 230 & $16.7 \pm 0.2$ & $39 \pm 0.4$ & 2015, July & (Nagai et al. 2016) \\
\hline $0923+392$ & XPOL & 260 & $6.1 \pm 2.3$ & $-52.59 \pm 10.97$ & 2016, January & NIKA2 - XPOL joint session \\
\hline
\end{tabular}

and

$\sigma_{\hat{p}}=\frac{\sqrt{Q^{2} \sigma_{Q}^{2}+U^{2} \sigma_{U}^{2}+\hat{p}^{4} I^{2} \sigma_{I}^{2}}}{\hat{p} I^{2}}$.

As far as the angle estimator is concerned, in the case of high $\mathrm{S} / \mathrm{N}$, we also reach the limit where the classical estimator $\psi=$ $1 / 2 \arctan (U / Q)$ is valid, with its associated uncertainty given by

$\sigma_{\psi}=\frac{\sqrt{Q^{2} \sigma_{q}^{2}+U^{2} \sigma_{u}^{2}}}{2(\hat{p} I)^{2}}$.

To express polarization angles, we use the IAU convention, which counts East from North in the equatorial coordinate system. The results obtained on the quasars observed are reported in Table 1 . Notice that the uncertainties in $Q$ and $U$ are generally comparable, as expected. Differences between the uncertainty values in $Q$ and $U$ of the quasar 3C 279 can be explained by residual correlated pixel-to-pixel noise. In addition, the absolute calibration uncertainty estimated at a level of $14 \%$ at $1.15 \mathrm{~mm}$ and $5 \%$ at $2.05 \mathrm{~mm}$ has to be added. These values come from the intensity flux dispersion measured on all scans of Uranus during an observational campaign. An additional uncertainty is linked to the HWP zero-position determination as discussed in Sect. 3.

We begin by comparing the NIKA results obtained in terms of degree and angle of polarization for the quasar 3C 286, which is considered a polarization calibrator at millimeter wavelengths, to those of XPOL (Agudo et al. 2012), SMA (Marrone 2006), CARMA (Hull \& Plambeck 2015), SMA (Hull et al. 2016), and ALMA (Nagai et al. 2016) experiments presented in Table 2 and Fig. 10. The results found by NIKA are consistent with those of XPOL within $1 \sigma$ error bar, whereas they are consistent within $2 \sigma$ error bars with the other experiments. Forthcoming observations with the NIKA2 instrument could improve the understanding of this discrepancy. The results obtained confirm that the quasar 3C 286 is highly polarized.

Similar results are found by comparing the NIKA data for 3C 279 with those of SHARP (Lee et al. 2015) at (857, $85.7,42.8,23) \mathrm{GHz}$. Lastly, we had the opportunity to observe $0923+392$ with XPOL in January 2016 during a test run of NIKA2 and found consistent results with our NIKA observations of this quasar taken in February 2015. Our measurements of polarization degrees and orientation therefore agree with other experiments on significantly polarized sources down to $\sim 60 \mathrm{mJy}$ (polarized flux) and polarization degree as low as $\sim 3 \%$. In order to ease the comparison of the results obtained by NIKA and other experiments we show them in Fig. 10.

\subsection{Noise equivalent flux density (NEFD) in polarization observations}

The NEFD gives an estimation of the sensitivity of the instrument per frequency band. It represents the uncertainty on the measure of the flux of point source in one second of integration. We must estimate the NIKA NEFD in both total intensity and polarization, and make sure that it is consistent with NIKA's sensitivity when used in total power mode only, as reported in Catalano et al. (2014), up to a factor two due to the presence of the analyzer after the HWP that rejects half the incident photons. During our observation run, the best observations we could use for this noise monitoring were $1 \mathrm{~h} 40 \mathrm{~m}$ of integration on $3 \mathrm{C} 286$. However, this is still a limited amount of time that leaves atmospheric residuals on our total intensity maps that prevents us from using them to derive NEFDs directly. However, with our HWP modulation, polarization is not affected by low-frequency atmospheric or electronic noise. The measured NEFDs in $Q$ and $U$ (equal to each other) are therefore more reliable. We find $120 \mathrm{mJy} \mathrm{s}^{1 / 2}$ at $260 \mathrm{GHz}$ and $50 \mathrm{mJy} \mathrm{s}^{1 / 2}$ at $150 \mathrm{GHz}$. Trusting these values, we can derive the expected NEFDs in $I$ that must be a factor $\sqrt{2}$ lower, that is to say $85 \mathrm{mJy} \mathrm{s}^{1 / 2}$ at $260 \mathrm{GHz}$ and $35.4 \mathrm{mJy} \mathrm{s}^{1 / 2}$ at $150 \mathrm{GHz}$. Now accounting for the expected factor two on absolute calibration (the NIKA primary calibrator is Uranus, which is unpolarized), due to the analyzer as mentioned before, we end up with $85 / 2=42.5$ and $35.4 / 2=17.7 \mathrm{mJy} \mathrm{s}^{1 / 2}$ at 260 and $150 \mathrm{GHz}$, respectively, in very good agreement with the measured values of 48 and 23 on NIKA in total power as reported in Catalano et al. (2014). Table 3 reports the summary of the NIKA polarimeter performance.

\subsection{Photometric accuracy}

The measurement of relatively stable quasars, such as 3C 286 and 3C 273, allows us to also cross check the quality of the NIKA photometry in intensity. Figure 11 presents the spectral energy density (SED) as a function of frequency in $\mathrm{GHz}$ for 3C 286 (left) and 3C 273 (right). The NIKA intensity flux and uncertainties at 1.15 and $2.05 \mathrm{~mm}$ are represented 

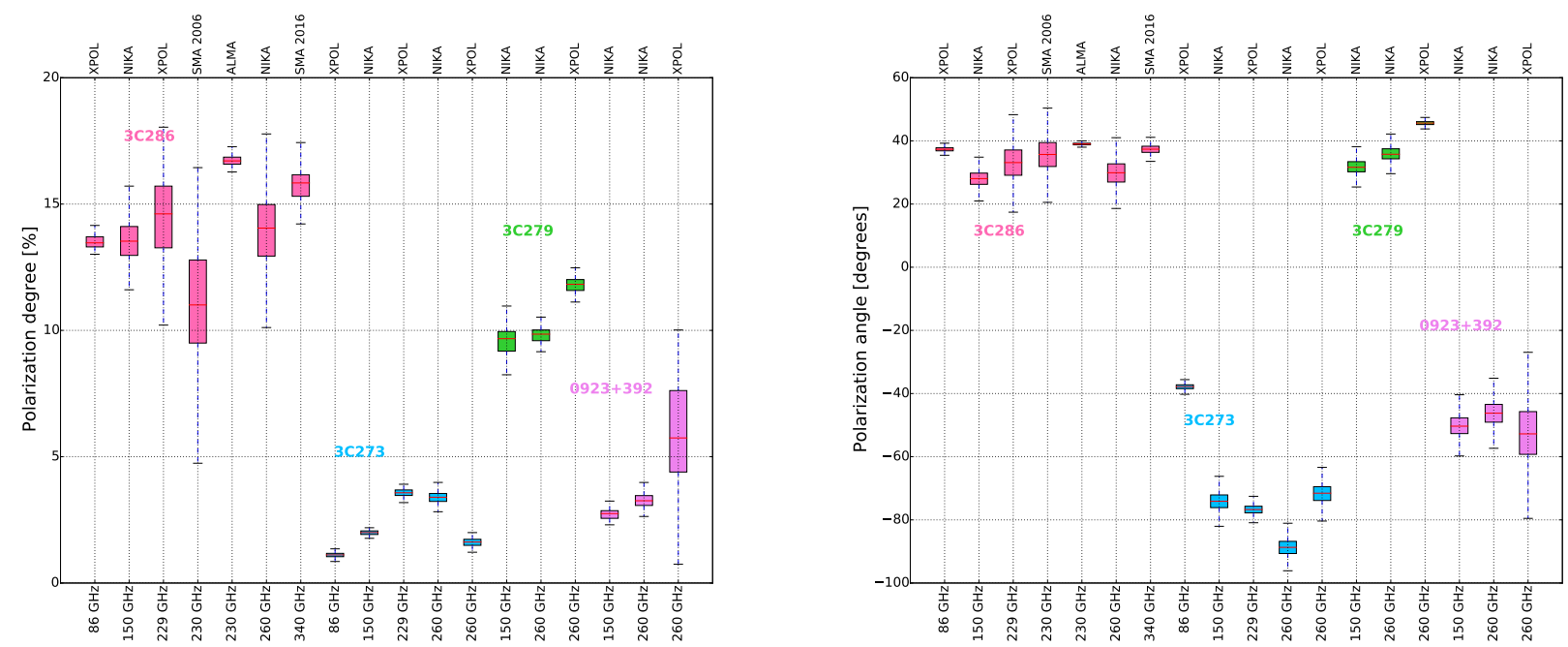

Fig. 10. Results on polarization degree (left) and angle (right) obtained by NIKA in comparison with other experiments. The values represented correspond to those reported in Tables 1 and 2. Dashed lines represent $2 \sigma$ error bars and the colored line in the box represents $1 \sigma$ error bars.
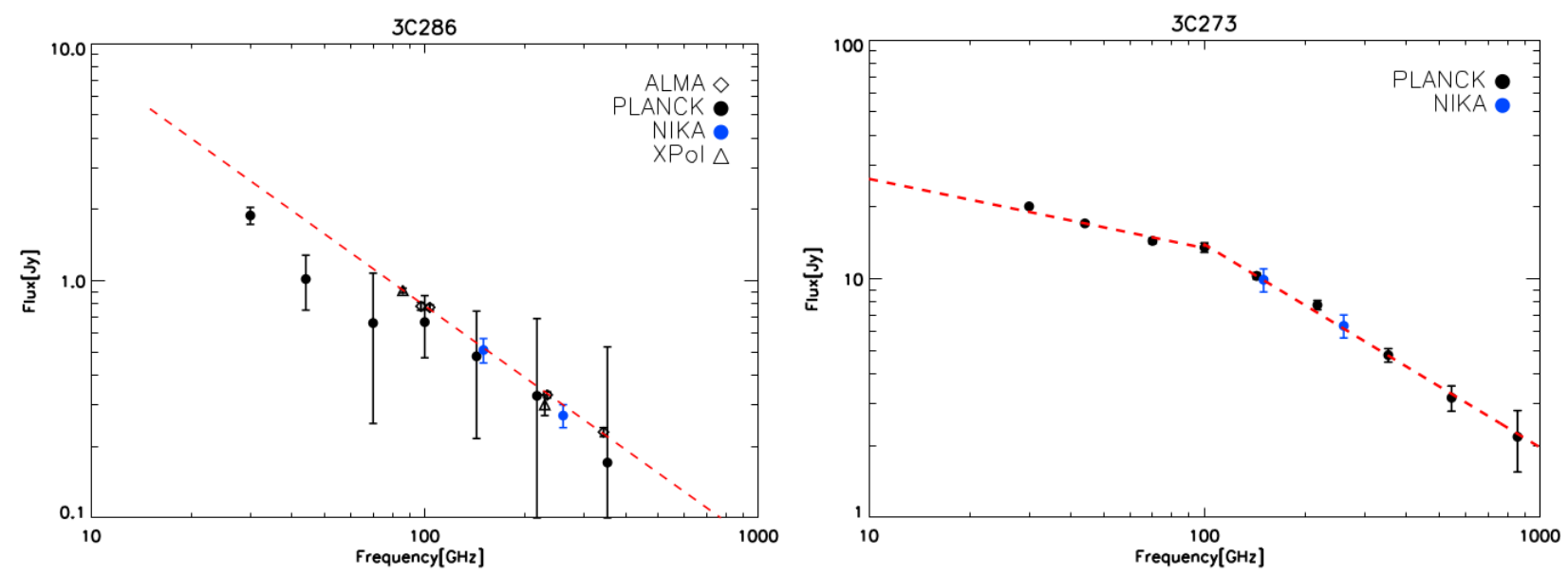

Fig. 11. SED for the quasars 3C 286 (left) and 3C 273 (right). We consider data from Planck (black dot, Planck Collaboration I 2014); ALMA (black diamond, Fomalont et al. 2014); XPOl (black triangle, Agudo et al. 2012) and NIKA (blue dot, this paper). For the fit, a color correction of $5 \%$ and $6 \%$ at $260 \mathrm{GHz}$ and $150 \mathrm{GHz}$, respectively, has been considered for both quasars.

Table 3. Performance of the NIKA polarimeter.

\begin{tabular}{lcc}
\hline \hline Array & $1.15 \mathrm{~mm}$ & $2.05 \mathrm{~mm}$ \\
\hline Valid pixels & 132 & 224 \\
Field of View (arcmin) & 1.8 & 1.8 \\
Band-pass $(\mathrm{GHz})$ & $190-310$ & $110-180$ \\
FWHM (arcsec) & 12 & 18.2 \\
Polarization capability & yes & yes \\
Sensit. on polarization $(Q)\left(\mathrm{mJy} \mathrm{s}^{1 / 2}\right)$ & 120 & 50 \\
Sensit. on $I$ in pol. mode $\left(\mathrm{mJy} \mathrm{s}^{1 / 2}\right)$ & 85 & 35 \\
Sensit. on $I$ in tot. power mode $\left(\mathrm{mJy} \mathrm{s}^{1 / 2}\right)$ & 42.5 & 17.7 \\
Instrumental polarization residual & $0.7 \%$ & $0.6 \%$ \\
Syst. uncertainty on pol. angle & $1.8^{\circ}$ & $1.8^{\circ}$ \\
\hline
\end{tabular}

in blue. Results from other experiments including XPOL (Thum et al. 2008), Planck (Planck Collaboration I 2014), and ALMA (Fomalont et al. 2014) are presented in black. We observe that the NIKA data are consistent within error bars with other experimental results. We find that $3 \mathrm{C} 286$ data are consistent with a synchrotron spectrum in the form of a power law, $\propto \nu^{\beta}$, with spectral index $\beta \simeq-1.007 \pm 0.033$ (dashed red line). To explain the 3C 273 data, we considered two power laws with spectral indices $\beta_{1} \simeq-0.29 \pm 0.05$ and $\beta_{2} \simeq-0.85 \pm 0.06$ (dashed red line) and knee frequency of $100 \mathrm{GHz}$.

\section{NIKA polarized observations of compact and extended sources}

We discuss here observations of compact and extended polarized sources, which allow us to further validate the quality of the reconstruction of the polarized sky signal with the NIKA camera. Special care is taken on the verification of the validity of the leakage correction algorithm, which can affect the reconstruction of the direction of polarization across the source. We have performed observations of different types of sources: Cygnus $\mathrm{A}$, a radio galaxy with diffuse emission between the two radio lobes; M 87, an external galaxy; and Orion OMC1, a nearby highly polarized galactic cloud. Observations of the Crab nebula have been performed several times during the NIKA polarization runs. A preliminary report on these observations has been given in Ritacco et al. (2016). A complete study of the polarized continuum emission observed at mm wavelengths for the Crab nebula is beyond the scope of this paper and will be included in a forthcoming publication (in prep.). 

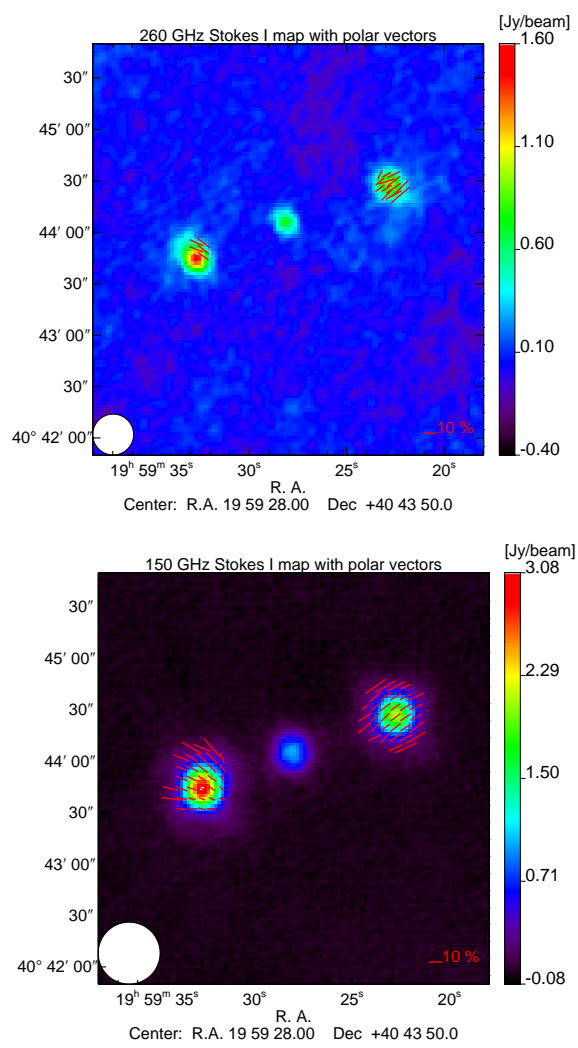
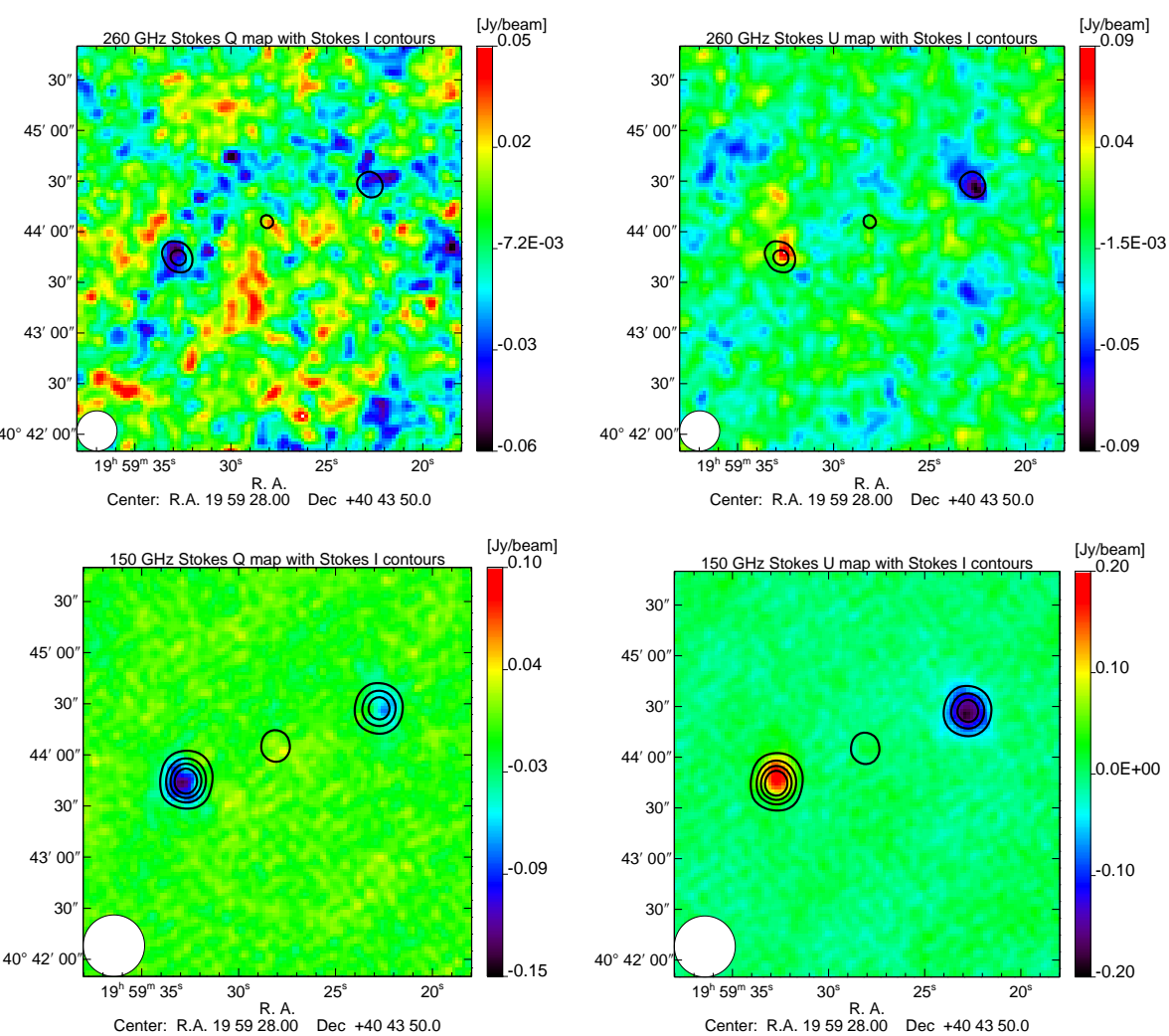

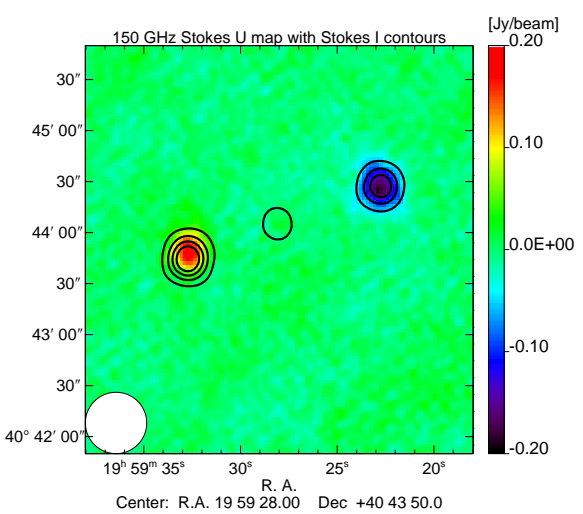

Fig. 12. Cygnus A Stokes $I, Q$, and $U$ maps at $260 \mathrm{GHz}$ (top) and $150 \mathrm{GHz}$ (bottom). The $Q$ and $U$ maps are smoothed with a Gaussian filter of 6 arcsec. At $260 \mathrm{GHz}$ the $I$ map is also smoothed to 4 arcsec for display purposes. The effective beam FWHM is shown as a white circle in the bottom left corner of each panel. The contours in $Q$ and $U$ represent the intensity map for each frequency. They start from $0.5 \mathrm{Jy} / \mathrm{beam}$ with steps of $0.5 \mathrm{Jy} /$ beam. Polarization vectors are plotted in red in the intensity image when $I>0$ and $P>2 \sigma_{\mathrm{P}}$.

\subsection{Cygnus $A$}

Cygnus $\mathrm{A}$ is a typical radio galaxy with twin jets of plasma emanating from its nucleus and forming two extended radio lobes. Cygnus $\mathrm{A}$ is the most powerful Fanaroff-Riley II (FRII) radio galaxy in the local environment. It has been well studied in terms of spatial resolution as it lies at a distance of $227 \mathrm{Mpc}$. At low radio frequencies, the synchrotron emission from the two giant lobes dominates (Hargrave \& Ryle 1974). At higher frequencies, the hotspots (working surfaces in the lobes) and the galaxy core become more prominent. The southern and northern hotspots are at 50 and 70 arcsec from the core, respectively. The complex structure of Cygnus A can be well explained by assuming that it consists of two components polarized in opposite directions and of an unpolarized core (Schraml \& Turlo 1967; Soboleva 1966; Mayer \& Hollinger 1968).

The NIKA Stokes $I, Q$, and $U$ maps of Cygnus A at 260 (top row) and 150 (bottom row) $\mathrm{GHz}$ are presented in Fig. 12. On the intensity maps, the polarization vectors are over-plotted in red. On the $Q$ and $U$ maps, intensity contours are also represented in black. As expected, on the intensity maps at 1.15 and $2.05 \mathrm{~mm}$, we clearly observe three compact sources that correspond to the core and the two hotspots. By contrast, the polarization maps show only two polarized regions that correspond to the hotspots and nothing on the core. This is an unambiguous confirmation of the astrophysical origin of the observed polarization: if it was due to the instrumental polarization, it would be proportional to the total intensity and therefore show up on the core. Such considerations are further confirmed on other extended sources as presented in the following sections.

\section{2. $M 87$}

M 87, also designated as 3C 274, or NGC 4486, or Virgo A, is a giant elliptical galaxy (de Vaucouleurs et al. 1976) located near the core of the Virgo cluster. Its nucleus is a radio and X-ray source from which emanates an optical jet. M 87 is estimated to be approximately $16 \mathrm{Mpc}$ from Earth (Mould et al. 1980). The core and the jet can be seen at all wavelengths from radio to X-rays. Figure 13 presents the NIKA Stokes $I, Q$, and $U$ maps at 260 (top) and 150 (bottom) GHz. The polarization vectors are over-plotted in red on the intensity maps representing both the degree and orientation of polarization. The peak surface brightness of the $I$ map is $\sim 0.6 \mathrm{Jy} /$ beam and $\sim 1.5 \mathrm{Jy} /$ beam at $260 \mathrm{GHz}$ and $150 \mathrm{GHz}$, respectively. Such a spectral index indicates that mm continuum emission in M 87 is indeed dominated by synchrotron emission. At $260 \mathrm{GHz}$ we do not have a significant detection of the polarization and the expected signal in $Q$ and $U$ maps is limited by the noise, as shown in the top panels of Fig. 13. However, the detection at $150 \mathrm{GHz}$ shows the polarization vectors well aligned following the intensity contours. They suggest the existence of a large-scale, ordered magnetic field in the radio lobes of $\mathrm{M} 87$.

\subsection{Orion $O M C-1$}

The Orion molecular cloud (OMC-1) is the closest site of OB star formation. The Nebula (KL) is the flux peak from far infrared to millimeter wavelengths on the OMC-1 "ridge" (Schleuning 1998). A sub-millimeter peak (KHW) with equal mass but lower dust temperature, is found 90 arcsec south of 

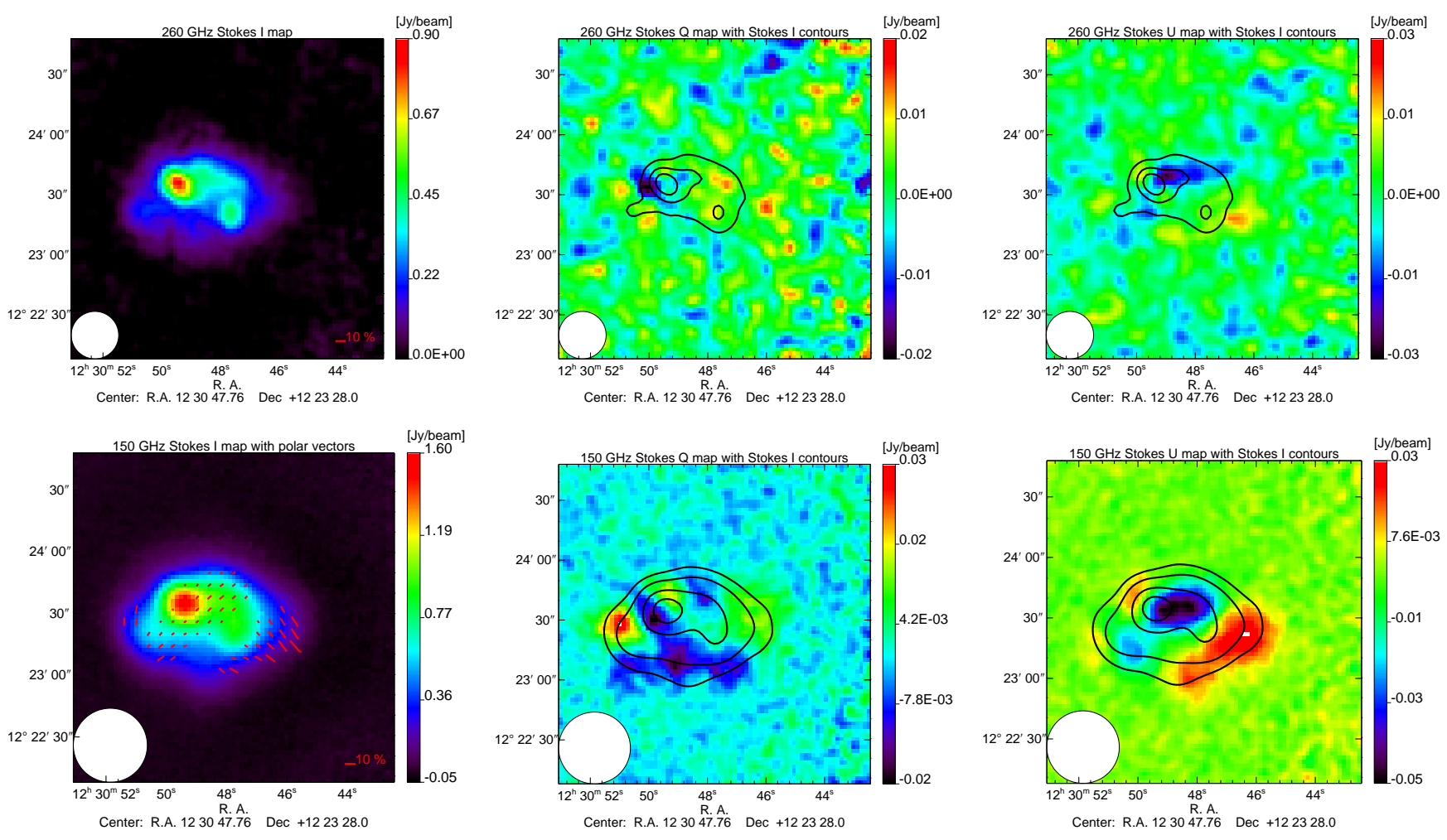

Fig. 13. NIKA M 87 Stokes $I, Q$, and $U$ maps at $260 \mathrm{GHz}$ (top) and $150 \mathrm{GHz}$ (bottom). For display purposes, at $260 \mathrm{GHz}$, the $Q$ and $U$ maps are smoothed with a Gaussian filter of 6 arcsec and the $I$ map of 4 arcsec. At $150 \mathrm{GHz}$ the $Q$ and $U$ maps are also smoothed to 4 arcsec, while the $I$ map is not smoothed. The contours in $Q$ and $U$ represent the intensity map for each frequency, starting from $0.2 \mathrm{Jy} / \mathrm{beam}$ with steps of $0.2 \mathrm{Jy} / \mathrm{beam}$. Polarization vectors are plotted in red in the intensity image when $I>0$ and $P>2 \sigma_{\mathrm{P}}$.

KL along the ridge (Keene et al. 1982). At KL and KHW, the polarization fraction increases with the wavelength (Schleuning 1998).

Figure 14 presents the NIKA Stokes $I, Q$, and $U$ maps of Orion OMC-1 at 260 (top) and 150 (bottom) GHz. Polarization vectors are over-plotted on the intensity maps, showing both the polarization degree and orientation. The peak surface brightness of the OMC-1 emission is approximately $45.8 \mathrm{Jy} /$ beam and $14 \mathrm{Jy} /$ beam at $260 \mathrm{GHz}$ and $150 \mathrm{GHz}$, respectively. The size of the map is $8 \times 8$ arcmin and is obtained by the co-addition of 18 maps for a total observational time of approximately $5 \mathrm{~h}$. The left panel of Fig. 15 shows the column density map obtained from the continuum emission of Orion OMC-1 observed at $1.15 \mathrm{~mm}$ assuming an average homogeneous dust temperature of $30 \mathrm{~K}$, see Lombardi et al. (2014). Mostly driven by our sensitivity limit, polarization is only detected at column densities $>3 \times 10^{23} \mathrm{~cm}^{-2}$ in the map. Depolarization is observed at column densities $>4 \times 10^{24} \mathrm{~cm}^{-2}$ at $1.15 \mathrm{~mm}$, while the $2.05 \mathrm{~mm}$ polarized fluxes seems less sensitive to depolarization.

The orientation of the polarization vectors is consistent between the 260 and $150 \mathrm{GHz}$ maps, confirming the same physical origin of the observed polarization. If we trust that the magnetic field is orthogonal to the direction of polarization, we observe a very organized magnetic field topology with field lines mostly oriented to the integral-shaped filament, and suggesting some bending of the field lines along the major axis of the filament towards the high-column-density cores. This magnetic field lines morphology could be due to the $\boldsymbol{B}$ dragged by largescale converging material accreting along the filament onto the core, or to the $\boldsymbol{B}$ being pushed by the powerful winds of the Orion nebula. These structures and filaments are consistent with
OMC-1 observations performed with SCUPOL (Matthews et al. 2009) at $850 \mu \mathrm{m}$. Previous observations at $1.3 \mathrm{~mm}$ performed by Leach et al. (1991) show that at the KL position, the average polarization rises to $4 \%-5 \%$ while at $\mathrm{KL}$, the polarization drops to $0.6 \%$.

The polarization intensity maps $P$ are reported on the top panel of Fig. 16a, showing the expected "polarization hole" across the KL nebula, already observed by Schleuning (1998). The polarization fraction maps $p$ as observed at both NIKA frequencies are reported on the central panel of Fig. 16b. On both maps, we observe a polarization fraction that reaches a level of approximately $10 \%$ of the total intensity in regions where the diffuse intensity emission is observed. This polarization fraction decreases greatly near the KL nebula. The polarization angle $\psi$ is shown on the bottom panel of Fig. 16c. Averaging across the KL nebula on 10 arcsec with central position $\alpha_{J 2000}: 05: 35: 14.098, \delta_{J 2000}:-05: 22: 31.00$ on the NIKA Orion maps at $260 \mathrm{GHz}(1.15 \mathrm{~mm})$ we find that the angle and degree of polarization are $\psi=(37.74 \pm 3.56)^{\circ}$ and $p=(0.6 \pm 0.2) \%$. The uncertainties reported here are purely statistical. We have also to consider the systematic uncertainties due to HWP zero position $1.8^{\circ}$ as well as the absolute calibration error calculated on Uranus, approximately $\sim 14 \%$ at $260 \mathrm{GHz}$ and $5 \%$ at $150 \mathrm{GHz}$. In Table 4, we summarize the results obtained on the KL region by SCUPOL and POLKA (Wiesemeyer et al. 2014) experiments for comparison with NIKA results.

\section{Conclusions}

This paper presents the first astrophysical polarization measurements with KIDs. For these measurements, we have adopted a 
A. Ritacco et al.: NIKA polarization performance
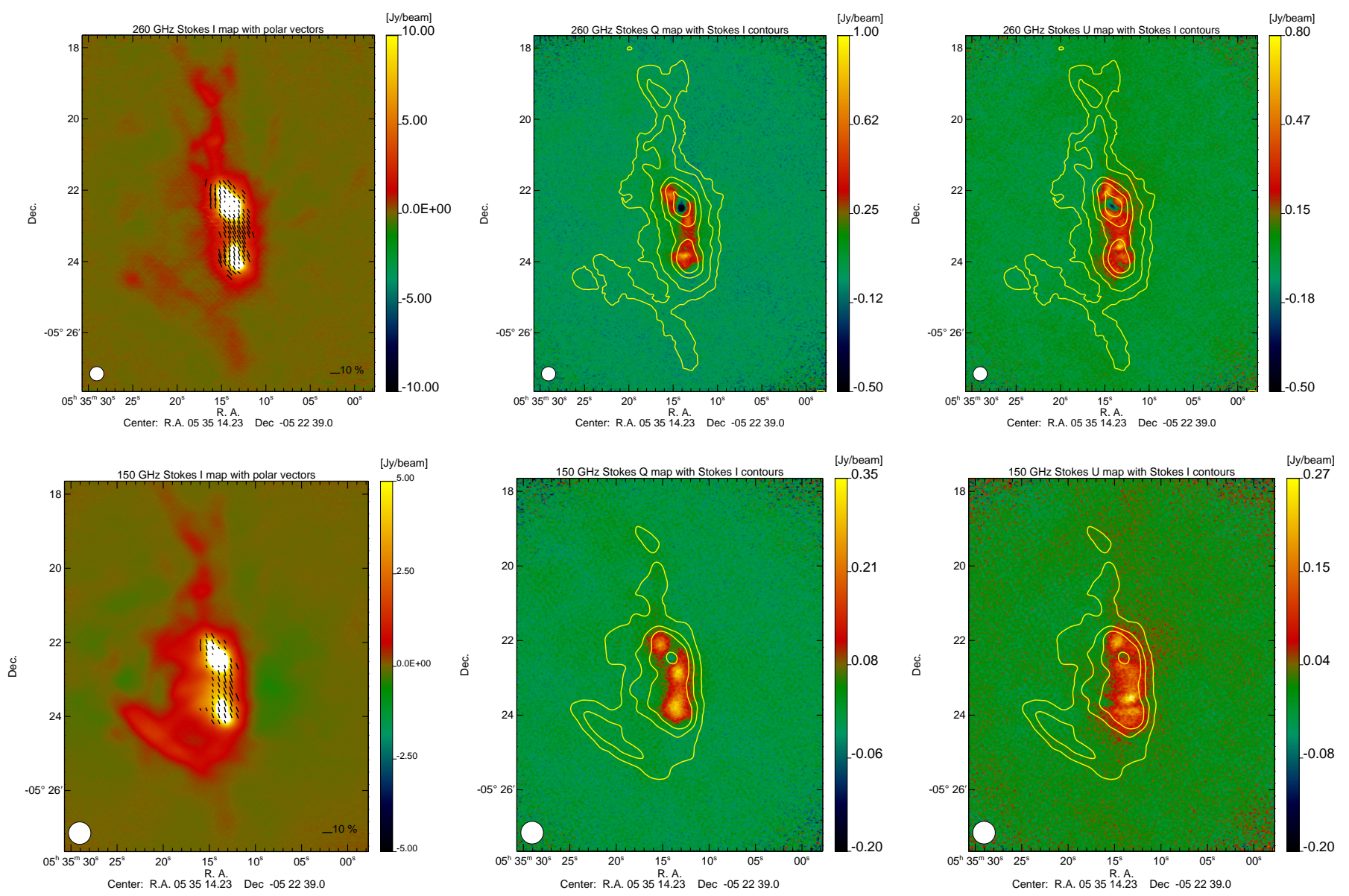

Fig. 14. NIKA Stokes $I, Q$, and $U$ maps of Orion OMC-1 at $260 \mathrm{GHz}$ (top) and $150 \mathrm{GHz}$ (bottom). The intensity contours over-plotted in the $Q$ and $U$ maps correspond to $(0.3,1,3,6,15$, and 48$) \mathrm{Jy} /$ beam at $260 \mathrm{GHz}$ and $(0.3,1,2,10,14) \mathrm{Jy} / \mathrm{beam}$ at $150 \mathrm{GHz}$. Polarization vectors are plotted in black in the intensity image when $I>0$ and $P>2 \sigma_{\mathrm{P}}$.
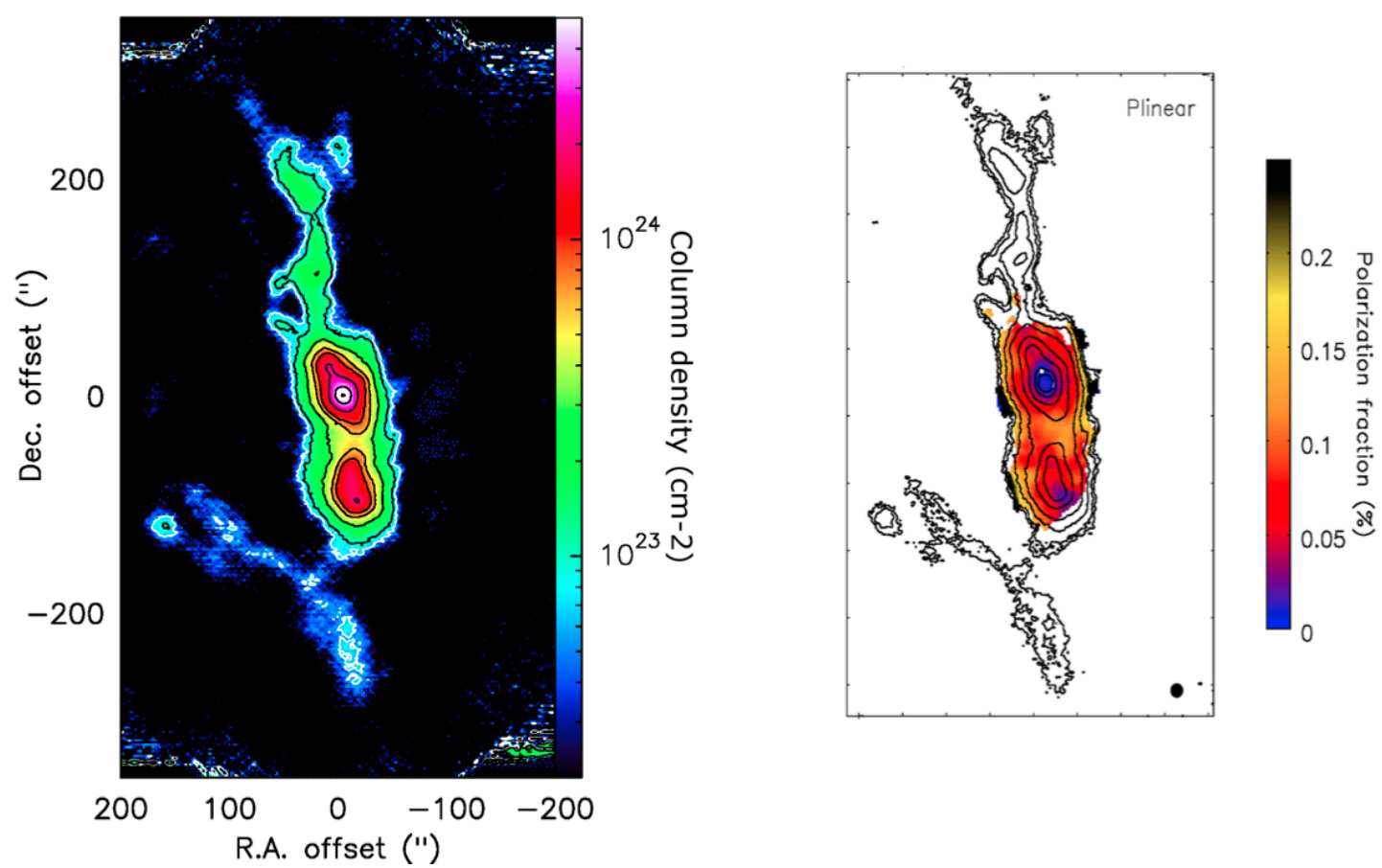

Fig. 15. Column density map (left) obtained from the intensity map $I$ at $1.15 \mathrm{~mm}$. For comparison the polarization fraction is reported on the right panel of the figure. 
Table 4. Summary of KL nebula polarization degree and angle results obtained by previous experiments and NIKA.

\begin{tabular}{cccccccc}
\hline \hline \multicolumn{7}{c}{$p[\%]$} & \multicolumn{7}{c}{$\psi\left[{ }^{\circ}\right]$} \\
\hline POLKA & SCUPOL & NIKA & NIKA & POLKA & SCUPOL & NIKA & NIKA \\
\hline$[870 \mu \mathrm{m}]$ & {$[850 \mu \mathrm{m}]$} & {$[1.15 \mathrm{~mm}]$} & {$[2.05 \mathrm{~mm}]$} & {$[870 \mu \mathrm{m}]$} & {$[850 \mu \mathrm{m}]$} & {$[1.15 \mathrm{~mm}]$} & {$[2.05 \mathrm{~mm}]$} \\
\hline $0.7 \pm 0.2$ & $0.7 \pm 0.1$ & $(0.6 \pm 0.2)$ & $(1.0 \pm 0.2)$ & $32.8 \pm 7.6$ & $40.8 \pm 5.4$ & {$[37.73 \pm 3.56]$} & {$[25.35 \pm 2.15]$} \\
\hline
\end{tabular}

Notes. An absolute uncertainty of $1.8^{\circ}$ has to be added to the statistical angle uncertainties reported here.

(a)
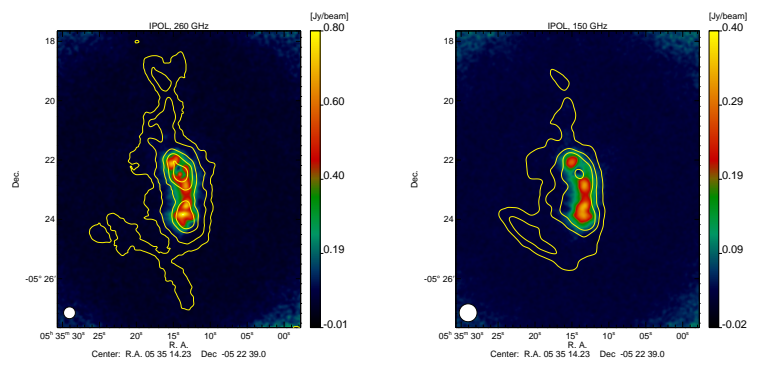

(b)
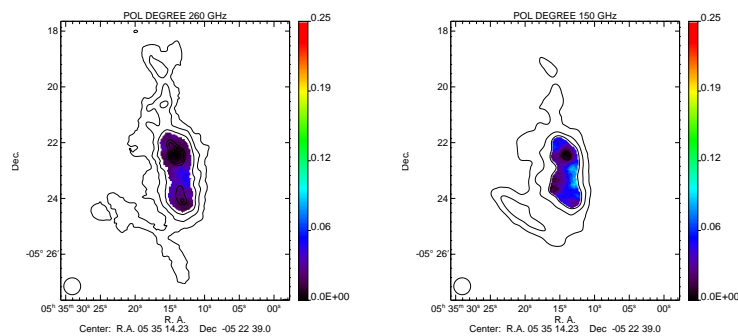

(c)
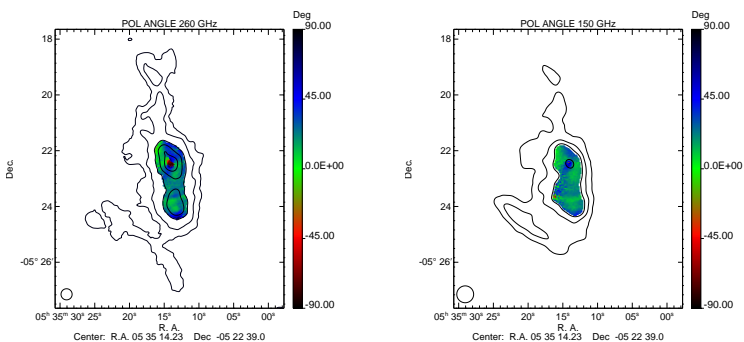

Fig. 16. Polarization intensity a); degree b) and angle c) maps of Orion Molecular Cloud (OMC-1) at $260 \mathrm{GHz}(l e f t)$ and $150 \mathrm{GHz}($ right $)$ with intensity contours over-plotted. Only the regions with $P>2 \sigma_{\mathrm{P}}$ are plotted on b) and c). At both frequencies, for display purposes, the polarization intensity maps are smoothed with a Gaussian filter of 6 arcsec; while the polarization degree and angle maps are smoothed to 2 arcsec.

simplified polarization system consisting of an achromatic, continuously rotating HWP at approximately $3 \mathrm{~Hz}$, an analyzer, and arrays of KIDs not sensitive to polarization. The fast modulation of the input polarization signal with the HWP allowed us to significantly reduce the atmospheric emission in the polarized signal. Instrumental polarization in the form of intensity to polarization leakage with a non-trivial-point-spread function has been observed at the level of $2 \%$ to $3 \%$ peak to peak for point- like and extended sources, respectively. We have successfully developed an algorithm to correct for this systematic effect. We are then left another kind of instrumental polarization that generates a polarized signal directly proportional to intensity at the level of $0.7 \%$ and $0.6 \%$ at 1.15 and $2.05 \mathrm{~mm}$, respectively, that can be corrected. We have observed $3 \mathrm{C} 286$, a quasar used as a standard polarization calibrator in the literature and have found a total flux, a polarization degree, and orientation in agreement with existing data. These results confirms findings for other quasars, such as 3C 279, 3C 273, and 0923+392, for which we either comparable results in the literature or performed simultaneous measurements with XPOL. We have also observed compact and extended sources such as Cygnus A, OMC-1, and M 87, and, again, found consistent results with existing polarization maps at approximately the same wavelength (e.g. OMC-1 Matthews et al. 2009). All these observations establish the accuracy of our system and analysis on astronomical sources with fluxes of approximately one Jansky and degrees of polarization as low as 3\%. On extended sources such as OMC-1, NIKA observations confirm that polarization vectors align well with the intensity structures indicating the presence of well ordered magnetic fields. To our knowledge, our observations of Cygnus A and M 87 are the first ones in polarization at millimetric wavelengths. NIKA has been a successful test-bench for the NIKA2 ${ }^{1}$ camera, which shares the same polarization system, although limited to the $260 \mathrm{GHz}$ channel. NIKA2 will observe the sky at the same frequencies with ten times more detectors and a FOV of 6.5 arcmin. The NIKA2 camera has been installed at the IRAM 30 meter telescope in Spain on October 2015 to start its commissioning phase for unpolarized observations. A polarization dedicated commissioning will follow, during which we will improve our understanding of the observed instrumental polarization and our ability to correct for it.

This paper shows the potentialities of an instrument based on KIDS and a fast and continuously rotating HWP to measure polarization, especially from the ground, where atmosphere is a nuisance, even more at low temporal frequencies and large angular scales. It opens the way to forthcoming observations with NIKA2 that will undoubtedly provide advances in the field of Galactic emission and interactions with the magnetic field.

Acknowledgements. We would like to thank the IRAM staff for their support during the campaigns. The NIKA dilution cryostat was designed and built at the Institut Néel. In particular, we acknowledge the crucial contribution of the Cryogenics Group, and in particular Gregory Garde, Henri Rodenas, Jean Paul Leggeri, and Philippe Camus. This work has been partially funded by the Foundation Nanoscience Grenoble, the LabEx FOCUS ANR-11-LABX-0013 and the ANR under the contracts "MKIDS", "NIKA" and ANR-15-CE31-0017. This work has benefited from the support of the European Research Council Advanced Grant ORISTARS under the European Union's Seventh Framework Programme (Grant Agreement No. 291294). We acknowledge fundings from the ENIGMASS French LabEx (R.A. and F.R.), the CNES post-doctoral fellowship program (R.A.), the CNES doctoral fellowship program (A.R.) and the FOCUS French LabEx doctoral fellowship program (A.R.).

\section{References}

Abdo, A. A., Ackermann, M., Ajello, M., et al. 2010, ApJ, 714, L73 Adam, R., Comis, B., Macías-Pérez, J. F., et al. 2014, A\&A, 569, A66 Adam, R., Comis, B., Macías-Pérez, J.-F., et al. 2015, A\&A, 576, A12 Adam, R., Comis, B., Bartalucci, I., et al. 2016, A\&A, 586, A122 Agudo, I., Thum, C., Wiesemeyer, H., et al. 2012, A\&A, 541, A111

1 http://ipag.osug.fr/nika2 
André, P., Di Francesco, J., Ward-Thompson, D., et al. 2014, Protostars and Planets VI, 27

Arzoumanian, D., André, Ph., Didelon, P., et al. 2011, A\&A, 529, L6

Barron, D., Keating, B., \& POLARBEAR Collaboration. 2013, in Am. Astron. Soc. Meet. Abstr., 221, 432.10

Benoît, A., Ade, P., Amblard, A., et al. 2004, A\&A, 424, 571

BICEP2 and Keck Array Collaborations, Ade, P. A. R., Aikin, R. W., et al. 2015, ApJ, 806, 206

Calvo, M., Roesch, M., Désert, F. X., et al. 2013, A\&A, 551, L12

Calvo, M., Benoît, A., Catalano, A., et al. 2016, J. Low Temp. Phys., 184, 816

Catalano, A., Calvo, M., Ponthieu, N., et al. 2014, A\&A, 569, A9

Catalano, A., Adam, R., Ade, P., et al. 2016, ArXiv e-prints [arXiv: 1605.08628]

Chapman, D., Aboobaker, A. M., Ade, P., et al. 2014, in Am. Astron. Soc. Meet. Abstr., 223, 407.03

Collett, E. 1992, Polarized light. Fundamentals and applications (New York: Dekker)

de Vaucouleurs, G., de Vaucouleurs, A., \& Corwin, J. R. 1976, in Second reference catalogue of bright galaxies (Austin: University of Texas Press)

Doyle, S., Naylon, J., Mauskopf, P., et al. 2008, in SPIE Conf. Ser., 7020

Essinger-Hileman, T., Kusaka, A., Appel, J. W., et al. 2016, Rev. Sci. Instr., 87, 094503

Fomalont, E., van Kempen, T., Kneissl, R., et al. 2014, The Messenger, 155, 19

Fraisse, A. A., Ade, P. A. R., Amiri, M., et al. 2013, J. Cosmol. Astropart. Phys., 4, 047

Galitzki, N., Ade, P. A. R., Angilè, F. E., et al. 2014, J. Astron. Instrum., 3, 1440001

Génova-Santos, R., Rubiño-Martín, J. A., Rebolo, R., et al. 2015, in Highlights of Spanish Astrophysics VIII, eds. A. J. Cenarro, F. Figueras, C. HernándezMonteagudo, J. Trujillo Bueno, \& L. Valdivielso, 207

Greaves, J. S., Holland, W. S., Jenness, T., et al. 2003, MNRAS, 340, 353

Hargrave, P. J., \& Ryle, M. 1974, MNRAS, 166, 305

Haykin, S. 2008, Communication systems (John Wiley \& Sons)

Hezareh, T., Wiesemeyer, H., Houde, M., Gusdorf, A., \& Siringo, G. 2013 A\&A, 558, A45

Hinderks, J. R., Ade, P., Bock, J., et al. 2009, ApJ, 692, 1221

Hull, C. L. H., \& Plambeck, R. L. 2015, J. Astron. Instrum., 4, 50005

Hull, C. L. H., Girart, J. M., \& Zhang, Q. 2016, ApJ, 830, 124

Johnson, B. R., Collins, J., Abroe, M. E., et al. 2007, ApJ, 665, 42

Keene, J., Hildebrand, R. H., \& Whitcomb, S. E. 1982, ApJ, 252, L11

Lazarian, A. 2009, in Cosmic Dust - Near and Far, eds. T. Henning, E. Grün, \& J. Steinacker, ASP Conf. Ser., 414, 482

Leach, R. W., Clemens, D. P., Kane, B. D., \& Barvainis, R. 1991, ApJ, 370, 257

Lee, S.-S., Petrov, L., Byun, D.-Y., et al. 2014, AJ, 147, 77

Lee, S.-S., Kang, S., Byun, D.-Y., et al. 2015, ApJ, 808, L26

Li, H., Dowell, C. D., Kirby, L., Novak, G., \& Vaillancourt, J. E. 2008, Appl. Opt., 47, 422

Lombardi, M., Bouy, H., Alves, J., \& Lada, C. J. 2014, A\&A, 566, A45
Madsen, K. K., Fürst, F., Walton, D. J., et al. 2015, ApJ, 812, 14 Marrone, D. P. 2006, Ph.D. Thesis, Harvard University

Marrone, D. P., \& Rao, R. 2008, in Millimeter and Submillimeter Detectors and Instrumentation for Astronomy IV, Proc. SPIE, 7020, 70202

Matthews, B. C., McPhee, C. A., Fissel, L. M., \& Curran, R. L. 2009, ApJS, 182, 143

Mayer, C. H., \& Hollinger, J. P. 1968, ApJ, 151, 53

Misawa, R., Bernard, J.-P., Ade, P., et al. 2014, in SPIE Conf. Ser., 9153, 91531

Molinari, S., Swinyard, B., Bally, J., et al. 2010, A\&A, 518, L100

Monfardini, A., Swenson, L. J., Bideaud, A., et al. 2010, A\&A, 521, A29

Monfardini, A., Benoit, A., Bideaud, A., et al. 2011, ApJS, 194, 24

Montier, L., Plaszczynski, S., Levrier, F., et al. 2015, A\&A, 574, A135

Mould, J., Aaronson, M., \& Huchra, J. 1980, ApJ, 238, 458

Nagai, H., Nakanishi, K., Paladino, R., et al. 2016, ApJ, 824, 132

Niemack, M. D., Ade, P. A. R., Aguirre, J., et al. 2010, in SPIE Conf. Ser., 7741, 77411

Pereyra, A., \& Magalhães, A. M. 2004, ApJ, 603, 584

Perley, R. A., \& Butler, B. J. 2013, ApJS, 206, 16

Pisano, G., Savini, G., Ade, P. A. R., Haynes, V., \& Gear, W. K. 2006, Appl. Opt., 45, 6982

Planck Collaboration I. 2011, A\&A, 536, A1

Planck Collaboration. 2013, VizieR Online Data Catalog: VIII/91

Planck Collaboration I. 2014, A\&A, 571, A1

Planck Collaboration VIII. 2014, A\&A, 571, A8

Planck Collaboration XIII. 2015, A\&A, 594, A8

Planck Collaboration XIX. 2015, A\&A, 576, A104

Planck Collaboration XXI. 2015, A\&A, 576, A106

Planck Collaboration Int. XXXIII. 2016, A\&A, 586, A13

Planck Collaboration Int. XLII. 2016, A\&A, 596, A103

Ritacco, A., Adam, R., Adane, A., et al. 2016, J. Low Temperature Phys., 184, 724

Roesch, M., Benoit, A., Bideaud, A., et al. 2012, ArXiv e-prints [arXiv: 1212.4585]

Savini, G., Pisano, G., \& Ade, P. A. R. 2006, Appl. Opt., 45, 8907

Schleuning, D. A. 1998, ApJ, 493, 811

Schleuning, D. A., Dowell, C. D., Hildebrand, R. H., Platt, S. R., \& Novak, G. 1997, PASP, 109, 307

Schraml, J., \& Turlo, Z. 1967, ApJ, 150, L15

Simmons, J. F. L., \& Stewart, B. G. 1985, A\&A, 142, 100

Simmons, J. F. L., Aspin, C., \& Brown, J. C. 1980, A\&A, 91, 97

Soboleva, N. S. 1966, Sov. Astron, 10, 214

Takahashi, Y. D., Ade, P. A. R., Barkats, D., et al. 2010, ApJ, 711, 1141

Thum, C., Wiesemeyer, H., Paubert, G., Navarro, S., \& Morris, D. 2008, PASP, 120,777

Wagner, J., Roy, A. L., Krichbaum, T. P., et al. 2015, A\&A, 581, A32

Wiesemeyer, H., Hezareh, T., Kreysa, E., et al. 2014, PASP, 126, 1027

Zhang, Q., Qiu, K., Girart, J. M., et al. 2014, ApJ, 792, 116 